Check for updates

Cite this: RSC Adv., 2019, 9, 40072

Received 21st September 2019

Accepted 19th November 2019

DOI: 10.1039/c9ra07653k

rsc.li/rsc-advances

\section{Synthesis of novel polycarbonyl Schiff bases by ring-opening reaction of ethyl 5-acyl-4-pyrone-2- carboxylates with primary mono- and diamines $\uparrow$}

\author{
D. L. Obydennov, iD * L. R. Khammatova, V. D. Steben'kov iD and V. Y. Sosnovskikh iD
}

An approach for the introduction of the tricarbonyl moiety into aromatic, heterocyclic, and aliphatic amines with the use of acylpyrones has been developed for the synthesis and the design of novel polycarbonyl Schiff base ligands, including salphen structures. This Michael addition-ring-opening reaction proceeds under mild conditions (stirring at $0-20^{\circ} \mathrm{C}$ ) via the attack at the C-6 position of the pyrone ring in good to high yields (up to $99 \%$ ) with excellent selectivity. The products can be easily isolated by crystallization without the use of chromatography. The scope of the reaction, tautomeric equilibrium of open-chain products, and their cyclization into pyridone structures were investigated.

\section{Introduction}

Polycarbonyl compounds are widely used in various fields of materials chemistry, analytical and inorganic chemistry due to their multifarious chemical properties ${ }^{\mathbf{1}}$ and complex-formation ability. ${ }^{2}$ Functional derivatives of 1,3-diketones, such as Schiff bases, play an especially important role as $N, N^{\prime}, O, O^{\prime}$-ligands and can give stable metal-chelate complexes with various metal ions. $^{3}$ These coordination compounds are actively used as catalytic systems for diverse organic reactions (synthesis of heterocycles $^{4 a-e}$ and polymerization ${ }^{4} f$ ), as well as chemosensors, ${ }^{5}$ materials for OLEDs, ${ }^{6}$ OFEDs, ${ }^{7}$ and solar cells. ${ }^{8}$

To date, although a large number of metal salen complexes have been studied, methods for further functionalization of the ligand structure remain important. The introduction of additional coordinating centers for the synthesis of multinucleating salphen complexes makes it possible to improve their catalytic, magnetic, optical properties and ion sensing. ${ }^{9}$

It is well known that the main method for the preparation of Schiff bases involves the condensation of carbonyl compounds with aromatic or aliphatic amines, ${ }^{\mathbf{1 0}}$ whereas the use of Michael reaction as an effective synthetic tool remains limited for the construction of ligands. ${ }^{11}$ Unlike the 1,3-dicarbonyl motif, the introduction of the polycarbonyl moiety into the molecule based on 1,3,5-triketones and other open-chain polyketones has some drawback, such as side reactions (the retro-Claisen cleavage, the intramolecular cyclization), a low selectivity, and low chemical reactivity of these substrates due to the presence of the acidic protons involved in the formation of the

Institute of Natural Sciences and Mathematics, Ural Federal University, 51 Lenina Ave., 620000 Ekaterinburg, Russian Federation. E-mail: dobydennov@mail.ru

$\uparrow$ Electronic supplementary information (ESI) available. See DOI: 10.1039/c9ra07653k intramolecular hydrogen bonds. ${ }^{12}$ In the literature there are only a few examples of salen ligand synthesis based on 2,4,6hexanetriones. ${ }^{13}$ Using acyl-4-pyrones as highly reactive synthetic equivalents of 1,3,5-triones, which can undergo Michael reaction under the action of amines as a key stage to circumvent the above difficulties. In this case, the reaction proceeds under mild conditions to avoid the formation of heterocyclization products and with high chemoselectivity. The ability of 4-pyrones to react with various $\mathrm{N}$-nucleophiles can allow the introduction of the polycarbonyl moiety in wide scope of substrates.

5-Acylcomanoates 1 (ref. 14) are convenient building blocks for the construction of heterocyclic systems via a nucleophilic attack at the C-6 position due to the presence of the electronwithdrawing substituent at the C-5 position followed by pyrone ring-opening (Fig. 1). Usually, 4-pyrones react with $\mathrm{N}$ nucleophiles via the ANRORC (Addition of the Nucleophile, Ring Opening, and Ring Closure) mechanism and give more stable azaheterocycles. ${ }^{14 a, b}$ Earlier, ${ }^{15}$ for the reaction of 2,5dicarbethoxy-4-pyrone and ethyl 5-benzoylcomanoate with $o$ phenylenediamine, the formation of polyfunctional open-chain polycarbonyl substrates only as intermediates in the synthesis of polycyclic pyridones have been shown.

In addition, in organic chemistry close attention is paid to the development of highly efficient synthetic methods, such as "Click Chemistry". This term describes reactions that are high yielding, wide in scope, stereospecific, simple to perform, high atom-economic, include facile the isolation of a product without the use of chromatography and can be carried out in easily removable or benign solvents. ${ }^{16}$ The search for such transformations is actively conducted, and Michael reaction and ring-opening reaction belong to a number of click-reactions. ${ }^{16}$ Pyrones can undergo the tandem of Michael reaction/ pyrone ring-opening, therefore, they are of interest for the 


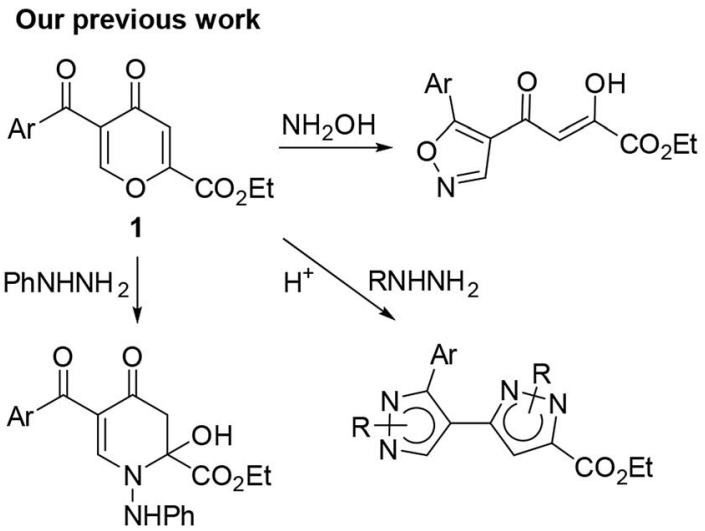

This work

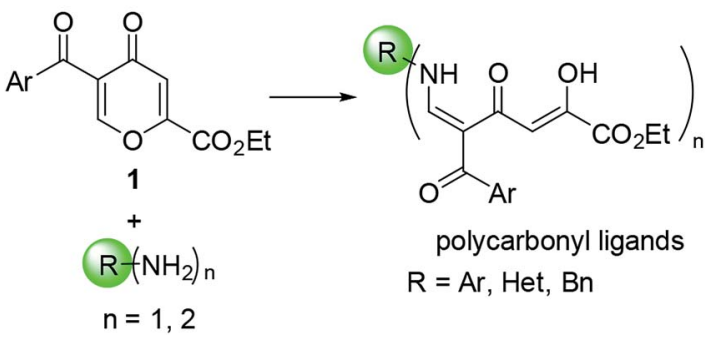

Fig. 1 Reactions of acylpyrones 1 with primary amines.

development of new synthetic click-approaches for the construction of polycarbonyl structures.

This work describes the general approach for the introduction of the 1,3,5-triketone moiety into the molecules bearing the amino group with the use of 5-acyl-4-pyrone-2-carboxylic acid esters to obtain and design new polycarbonyl schiff base ligands, including salphen structures, as well as to study their tautomeric equilibrium and the substrate scope of this method.

\section{Results and discussion}

We started the study with the reaction of 5-acylcomanoates 1 with various aromatic mono- and diamines 2 in $\mathrm{EtOH}$ at $0{ }^{\circ} \mathrm{C}$ or $20^{\circ} \mathrm{C}$ (Scheme 1 and Table 1). It was found that pyrones 1 gave tetracarbonyl enaminones 3a-o in medium to high yields (47$96 \%$ ). The nature of the substituents in the aromatic amine has a slight effect on the outcome of the reaction though unreactive amines bearing the electron-withdrawing group, such as 2aminopyridine and 2-aminopyrimidine, gave enaminones $3 \mathbf{k}$ and $3 \mathrm{~m}$ at $20^{\circ} \mathrm{C}$ in good yields ( $74 \%$ and $47 \%$, respectively). The transformation was found to be tolerant to steric hindrance of a bulky acyl group and, for pivaloylpyrone 1f bearing the bulky tert-butyl group, product $3 \mathbf{i}$ was obtained in $61 \%$ yield.

It was found that the polycarbonyl moiety can be introduced into the molecule of aliphatic amine, such as benzylamine (2h). Indeed, enaminones $3 \mathbf{n}, \mathbf{o}$ were obtained in lower yield (43-54\%) compared to aromatic amines. This result can be explained by easier intramolecular cyclization of enaminone 3 into stable pyridones because of higher nucleophilicity of the alkylamine.



Scheme 1 Reaction of pyrones 1 with amines.

Products 3 are multifunctionalized structures bearing the enaminodione and diketone moieties, which can undergo ketoenol tautomerism and $Z / E$ isomerization as the result of the push-pull nature of the double bond. Two geometric isomers $Z$ 3 and $E-3$ and also tautomer keto- $E-3$, which exist in equilibrium in the solution of $\mathrm{CDCl}_{3}$, were detected by NMR spectroscopy (Scheme 1 and Table 1).

A clear influence of the substituents on the tautomeric equilibrium is observed, which can be explained by the stability of the hydrogen bond formed and steric interactions. The introduction of the electron-donating $\mathrm{MeO}$ group into the aroyl substituent of $N$-phenyl-substituted enaminones 3a increases the content of the most stable isomer $E-3$ from $82 \%$ to $95 \%$. Although a change in the structure of the amine has an effect on the observed equilibrium, the introduction of the ortho substituent reduces the content of major tautomer $E-3$ to $76-$ $79 \%$. Enaminones $\mathbf{3 h}, \mathbf{j}, \mathbf{l}, \mathbf{m}$, which bear the thenoyl substituent, are especially distinguished in this series because the content of the major isomer $E-3$ reaches $87-93 \%$.

The structure of tautomer $E-3$ was confirmed on the basis of the coupling constants extracted from the ${ }^{1} \mathrm{H}$-coupled ${ }^{13} \mathrm{C}$ NMR spectrum of $3 \mathbf{b}$, along with the analysis of ${ }^{1} \mathrm{H}^{13} \mathrm{C}$ HMBC spectrum of $3 \mathbf{b}$. It is known ${ }^{17}$ that, in the case of enones, coupling constant ${ }^{3} J_{\mathrm{H}, \mathrm{C}}$ between the $\beta$-olefinic protons and the carbonyl group strongly depends on the dihedral angle and is a good criterion for establishing the configuration of the double bond when other experiments cannot be applied. For NCsubstituted enaminones, the $\mathrm{CH}$ proton of the $\mathrm{N}-\mathrm{CH}=$ moiety has ${ }^{3} \mathrm{~J}_{\mathrm{H}, \mathrm{CN}}$ coupling with the cyano group in the range of 4.6-5.8 Hz and 9.6-10.8 Hz for their cis- and trans-orientation, respectively. ${ }^{18}$ Also in the literature the ${ }^{3} \mathrm{~J}_{\mathrm{H}, \mathrm{C}=\mathrm{O}}$ coupling has been measured $^{19}$ for similar structures, alkoxymethylidenediones $\left(\right.$ cis $^{-3}{ }_{\mathrm{H}, \mathrm{C}=\mathrm{O}}=2.6-4.0 \mathrm{~Hz}$, $\operatorname{trans}^{-}{ }^{3} \mathrm{~J}_{\mathrm{H}, \mathrm{C}=\mathrm{O}}=$ 7.7-10.1 Hz) and the magnitudes of these ${ }^{3} J_{\mathrm{H}, \mathrm{C}=\mathrm{O}}$ coupling 
Table 1 Reaction scope study of pyrones 1 with amines $2^{a}$



\footnotetext{
${ }^{a}$ Pyrone $1(1.11 \mathrm{mmol})$ and amine $2(1.11 \mathrm{mmol})$ were stirred in EtOH $(6 \mathrm{~mL})$ at $0{ }^{\circ} \mathrm{C}$ for $1 \mathrm{~h} .{ }^{b}$ The reaction was performed at $20{ }^{\circ} \mathrm{C}$ for $2 \mathrm{~h} .{ }^{c}$ The reaction was performed at $20^{\circ} \mathrm{C}$ for 4 days, $2.22 \mathrm{mmol}$ of the amine was used.
}

constants are convenient parameters for establishment of enaminodione configuration in our case. It was found that for enaminodione $\mathbf{3 b}$ the carbonyl group of the aroyl substituent $\left(\mathrm{C}-4^{\prime}\right)$ is a quartet at $\delta 193.0 \mathrm{ppm}$ with ${ }^{3} J_{\mathrm{H}, \mathrm{C}}=3.7 \mathrm{~Hz}$, which indicates the cis-arrangement of the $\mathrm{H}-6$ proton relative to C- $4^{\prime}$ (Fig. 2). The signal for the carbonyl group of the diketone moiety (C-4) in the spectrum of $\mathbf{3 b}$ appears as a doublet of doublets and exhibits a ${ }^{3} J_{\mathrm{C}-\mathrm{H}}$ coupling of $7.8 \mathrm{~Hz}$ with the $\mathrm{H}-6$ proton and a small ${ }^{2} J_{\mathrm{C}-\mathrm{H}}$ coupling of $3.7 \mathrm{~Hz}$, which is in consistent with the magnitude of the coupling constant ${ }^{2} J_{\mathrm{C}-\mathrm{H}}$ in the $=\mathrm{CH}-\mathrm{C}=\mathrm{O}$

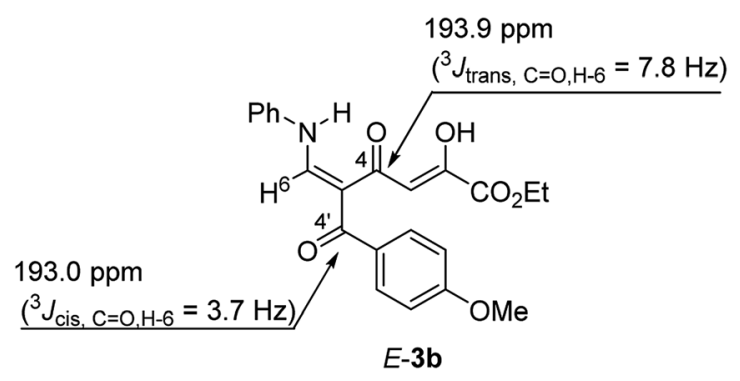

Fig. 2 Selected ${ }^{13} \mathrm{C}$ NMR signals of $E-3 b$. moiety $(2.3-3.3 \mathrm{~Hz}) .^{20}$ The value of the first $(7.8 \mathrm{~Hz})$ is more than ${ }^{3} J_{\mathrm{H}, \mathrm{C}}$ for $\mathrm{C}-4^{\prime}(3.7 \mathrm{~Hz})$ and is in agreement with the transarrangement of $\mathrm{H}-6$ and C-4, which corresponds structure $E-3 \mathbf{b}$.

The ${ }^{1} \mathrm{H}$ NMR spectra of polycarbonyl compounds (het)aryl substituted 3a-m feature a main set of signals of tautomer $E-3$ with the $5 E$-configuration of the double bond which consists of a doublet of the NH proton at $\delta 12.07-12.60 \mathrm{ppm}\left({ }^{3} J=12.4-13.6\right.$ $\mathrm{Hz})$ and a downfield singlet for the OH group $(\delta$ 14.27-14.50 $\mathrm{ppm})$, apparently involved in a strong intramolecular hydrogen bonds, a doublet of the $=\mathrm{CHN}$ proton at $\delta 7.86-8.81 \mathrm{ppm}\left({ }^{3} \mathrm{~J}=\right.$ $12.4-13.6 \mathrm{~Hz}$ ), and a vinylic proton singlet at $\delta 6.59-7.15 \mathrm{ppm}$. For Bn-substituted $3 \mathbf{n}, \mathbf{o}$, the signals of both the $=\mathrm{CHN}$ proton and the $\mathrm{NH}$ proton are shifted upfield compared with those of (het)aryl substituted 3a-m and appeared at $\delta$ 7.49-7.69 and $10.95 \mathrm{ppm}$, respectively.

The characteristic signals of minor tautomer $Z-3$ with $5 Z$ configuration include a doublet of the $=$ CHN proton $(\delta 8.27-$ $9.30 \mathrm{ppm},{ }^{3} J=12.8-14.0 \mathrm{~Hz}$ ), which is shifted downfield by $c a$. $0.5 \mathrm{ppm}$ compared with the same proton of $E-3$, a singlet for the $=\mathrm{CHCO}$ proton $(\delta 5.84-6.34 \mathrm{ppm})$ is shifted upfield by $0.7-$ $1.2 \mathrm{ppm}$ compared to $E-3$ isomer due to a shielding influence of the aromatic ring of the acyl moiety (Fig. 3). Such a difference in 


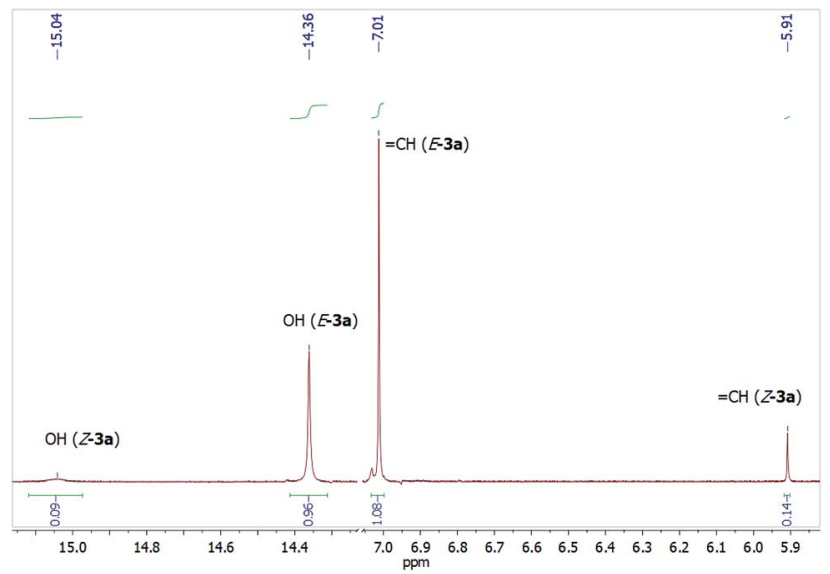

Fig. 3 Fragment of the ${ }^{1} \mathrm{H}$ NMR spectrum of $3 a$ in $\mathrm{CDCl}_{3}$.

the chemical shifts of these protons was not observed for pivaloyl-substituted enaminones $\mathbf{3 i}, \mathbf{n}$ (only $0.01-0.2 \mathrm{ppm}$ ). The $\mathrm{OH}$ proton and the $\mathrm{NH}$ proton appear as broadened signals at $\delta$ 15.0-15.4 ppm and $\delta 11.19-12.60 \mathrm{ppm}$ (for aryl substituted 3a$\mathbf{m}, \delta$ 10.04-10.95 ppm for benzyl substituted 3n,o), respectively, as a result of the formation of the intramolecular hydrogen bonds. It is important to note that the $\mathrm{OH}$ proton signal is shifted downfield by $c a .0 .8 \mathrm{ppm}$ in comparison with the same proton of $E-3$ isomer, which indicates the stronger intramolecular hydrogen bond $(\mathrm{OH} \cdots \mathrm{O}=)$ in the $Z-3$ isomer and is consistent with the proposed structures.

It is well known ${ }^{14 b, d}$ that diketobutanoates predominantly exist as the enol form and contain some quantity of the diketo form $(0-10 \%)$. Therefore, the structure of keto- $E-3$ was established on the basis of the relative content of the tautomer (0$13 \%)$. In ${ }^{1} \mathrm{H}$ NMR spectra the feature signals of the tautomer are a doublet $\left({ }^{3} J \approx 13.0 \mathrm{~Hz}\right)$ of the downfield $\mathrm{NH}$ proton at $\delta 12.22-$ $12.60 \mathrm{ppm}$ (for keto- $E-3 \mathrm{a}-\mathrm{m}$ ) or $\delta 11.23-11.25 \mathrm{ppm}$ (for keto- $E$ $3 \mathbf{n}-\mathbf{o})$ and a singlet of the $\mathrm{CH}_{2}$ group at $\delta 4.07-4.40 \mathrm{ppm}$. The signal of the NH proton of the tautomer is shifted downfield by 0.2-0.6 ppm compared to the same proton of $E-3$ isomer because of stronger electron-withdrawing properties of the nonconjugated carbonyl group of the diketone moiety.

Enaminones $\mathbf{3}$ are of interest as chemically active substances for further transformations. When substrates 3 bearing the amino group react with pyrone $\mathbf{1}$ in EtOH at room temperature for $24 \mathrm{~h}$, they give polycarbonyl ligands $4 a-e$ in $64-79 \%$ yields (Method A, Scheme 2 and Table 2), which are yellow or red precipitates and stable during storage in the solid state. Polycarbonyl ligands 4 can also be obtained directly from $o$-phenylenediamines 2 by the reaction with two equivalents of 4pyrones 1 in $31-63 \%$ yields (Method B).

Theoretically, compounds $\mathbf{4}$ can be represented as ten openchain tautomeric structures, but in the ${ }^{1} \mathrm{H}$ NMR spectra it is possible to detect and assign some signals only for $5 E, 5^{\prime} E-4$ and $5 E, 5^{\prime} Z-4$ tautomers by comparing NMR signals of parent polycarbonyl compounds 3 .

The structure of compounds 4 was confirmed on the basis of elemental analysis data, IR and NMR spectra. In ${ }^{1} \mathrm{H}$ NMR spectra in $\mathrm{CDCl}_{3}$ major isomer $5 E, 5^{\prime} E-4$ appeared as a singlet of the $\mathrm{H}-3$ proton at $\delta 6.82-6.95 \mathrm{ppm}$, doublets of the $\mathrm{H}-6$ proton and the NH group at $\delta 7.92-8.09 \mathrm{ppm}$ and $12.41-12.58 \mathrm{ppm}\left({ }^{3} \mathrm{~J}=\right.$ 12.4-12.6 Hz), respectively. The characteristic signals of minor isomer $5 E, 5^{\prime} Z-4$ include two singlets at $\delta 5.90-6.36$ and 6.41$6.45 \mathrm{ppm}$ attributed to the vinylic protons of the diketone motifs and two doublets of the $=\mathrm{CHN}$ protons at $\delta 7.99$ $8.12 \mathrm{ppm}$ and 8.36-8.53 ppm.

It was found that the content of the most stable $5 E, 5^{\prime} E-4$ tautomer is equal to $39-74 \%$. The nature of the substituents in the aroyl group has a strong influence on the content of this tautomer. In the case of electron-donating substituents, the content of $5 E, 5^{\prime} E$-isomer is the highest $(69-74 \%$ for $\mathbf{4 b}, \mathbf{d}, \mathbf{e})$, while the introduction of the electron-withdrawing substituent $\left(\mathrm{Ar}=4-\mathrm{ClC}_{6} \mathrm{H}_{4}\right)$ leads to a decrease in the content of the main isomer to $39 \%$. These data are also consistent with the amount

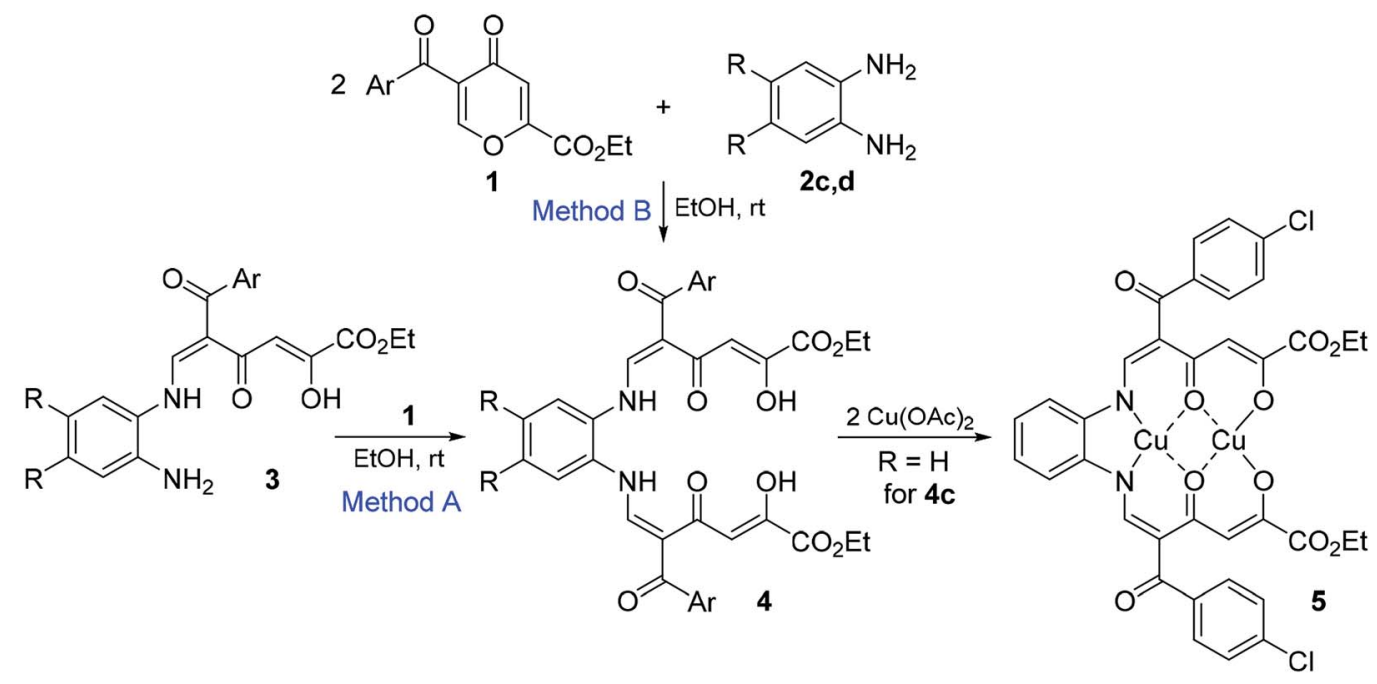

Scheme 2 Reactions of pyrones 1 with diamines 2 . 
Table 2 Scope of the pyrone transformation with diamines $2^{a}$

\begin{tabular}{|c|c|c|c|c|c|}
\hline Bis-adduct & $\mathrm{Ar}$ & $\mathrm{R}$ & $\begin{array}{l}\text { Yield from enaminone } 3 \text {, } \\
\%(\text { Method } \mathrm{A})\end{array}$ & $\begin{array}{l}\text { Yield from diamine, } \\
\% \text { (Method B) }\end{array}$ & $5 E, 5^{\prime} E-4: 5 E, 5^{\prime} Z-4$ \\
\hline $4 a$ & $\mathrm{Ph}$ & $\mathrm{H}$ & 64 & 57 & $50: 17$ \\
\hline $4 c$ & $4-\mathrm{ClC}_{6} \mathrm{H}_{4}$ & $\mathrm{H}$ & 65 & 75 & $39: 26$ \\
\hline 4d & 2-Th & $\mathrm{H}$ & 75 & 31 & $71: 14$ \\
\hline $4 e$ & $2-\mathrm{Th}$ & $\mathrm{Me}$ & 79 & 63 & $74: 10$ \\
\hline
\end{tabular}

${ }^{a}$ Method A: pyrone $1(0.26 \mathrm{mmol})$ and enaminone $3(0.26 \mathrm{mmol})$ were stirred in EtOH $(2 \mathrm{~mL})$ at $20{ }^{\circ} \mathrm{C}$ for $24 \mathrm{~h}$. Method B: pyrone $1(0.72 \mathrm{mmol})$ and diamine $2(0.36 \mathrm{mmol})$ were stirred in $\mathrm{EtOH}(2 \mathrm{~mL})$ at $0{ }^{\circ} \mathrm{C}$ for $1 \mathrm{~h}$ and then at $20^{\circ} \mathrm{C}$ for $24 \mathrm{~h}$.<smiles>CCOc1cc(=O)c(C(=O)c2ccccc2)co1</smiles><smiles>CCOC(=O)/C=C(\O)C(=O)/C(=C/Nc1ccc(N/N=C/C(=O)C(=O)c2ccccc2)cc1)C(=O)O</smiles>

Scheme 3 Reaction of pyrone 1a with p-phenylenediamine.

of minor $5 E, 5^{\prime} Z$-isomer (10-26\%), the largest content (26\%) was found for $4 \mathbf{c}\left(\mathrm{Ar}=4-\mathrm{ClC}_{6} \mathrm{H}_{4}\right)$.

Compounds 4 are polydentate salphen structures, which makes them promising ligands for the formation of binuclear complex compounds with various metal ions, which have been recently received much attention for various application from catalysis to materials chemistry. ${ }^{9}$ It was found that compound 4c upon treatment with copper(II) acetate gives binuclear chelate complex $\mathbf{5}$, whose structure is in agreement with the literature data ${ }^{13}$ and is confirmed by elemental analysis, ESI-MS data, NMR and $\mathrm{IR}^{21}$ spectroscopies. ${ }^{1} \mathrm{H}$ NMR spectra for $\mathrm{Cu}(\mathrm{II})-$ coordinated compounds are usually not informative as the result of strong signal peak broadening and unpredictable chemicals shifts due to paramagnetic properties of $\mathrm{Cu}(\mathrm{II})$ compounds. $^{22}$ In contrast, binuclear complexes can produce narrow ${ }^{1} \mathrm{H}$ NMR resonance because of magnetic interaction of two paramagnetic copper(II) centers. ${ }^{22}$ The ${ }^{1} \mathrm{H}$ NMR spectrum of compound $\mathbf{5}$ appears to be useful and features a single set of signals, which consists of a broadened triplet for the Me group at $\delta 1.10 \mathrm{ppm}$, broadened quartet at $\delta 3.76 \mathrm{ppm}$, a broadened singlet for $=\mathrm{CH}$ proton at $\delta 5.74 \mathrm{ppm}$, and a multiplet for the aromatic protons at $\delta 7.24-7.80 \mathrm{ppm}$.

It is interesting to note that the reaction of benzoylpyrone $\mathbf{1 a}$ ( 2 equiv.) with $p$-phenylenediamine ( $2 \mathbf{i}, 1$ equiv.) proceeds very readily to form compound 6 in almost quantitative yield (99\%) (Scheme 3). Such a large difference in reactivity compared to $o$ phenylenediamine can be explained by less steric hindrances during the formation of the polycarbonyl ligands. In contrast to aromatic diamines, the reaction of pyrone 1a with ethylenediamine led to a product, which exists in solution as a complex mixture of tautomers.

The main drawback of the polycarbonyl Schiff base ligands bearing the triketone moiety is their ability to cyclize into pyridones, which may limit their application. Therefore, further we decided to study the chemical properties of the polycarbonyl compounds 3 , which are connected with their stability and the possibility of further modification for the design of new ligands.

Enaminones $\mathbf{3 e}, \mathbf{f}, \mathbf{h}, \mathbf{j}$ bearing the amino group in the ortho position can undergo intramolecular cyclization to form more

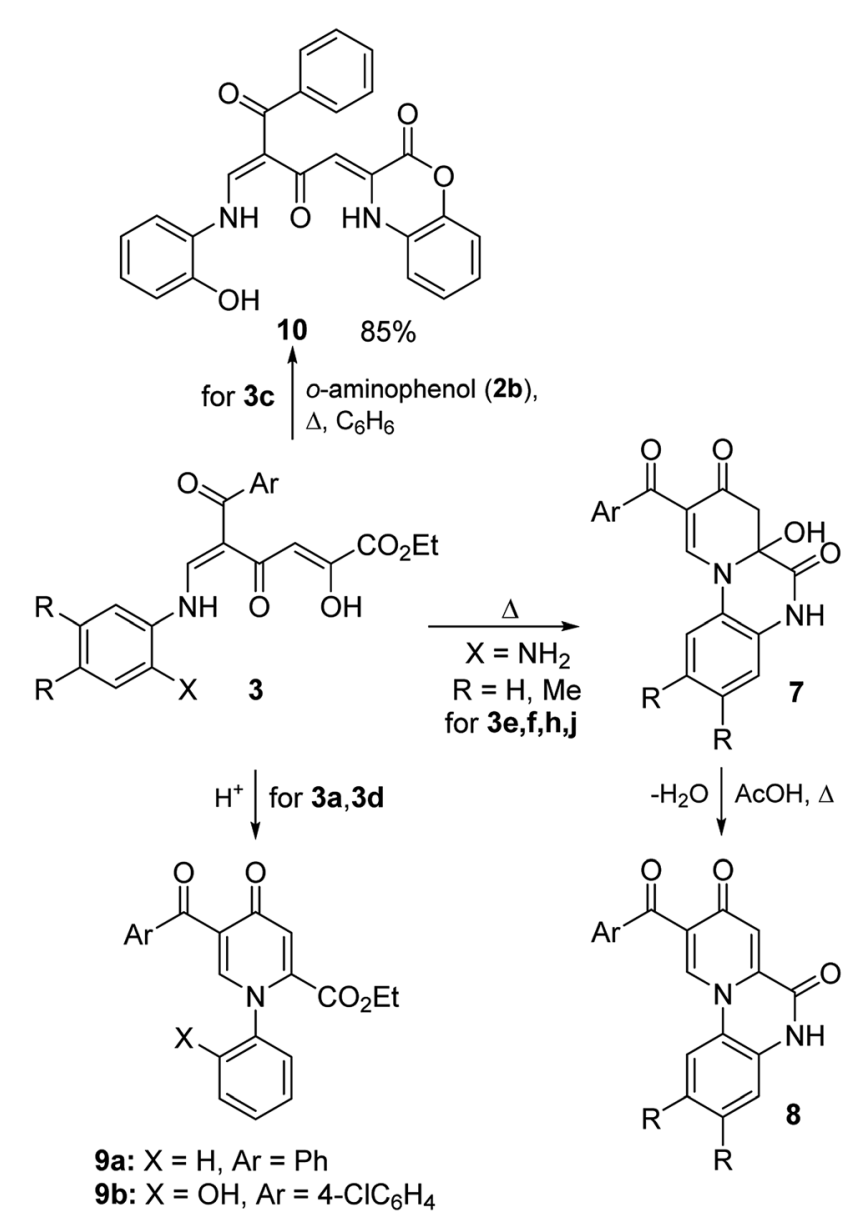

Scheme 4 Cyclization reactions of polycarbonyl compounds 3. 
Table 3 Synthesis of pyrido[1,2-a]quinoxaline-6,8-diones 7,8

\begin{tabular}{|c|c|c|c|c|c|c|}
\hline Starting enaminone & $\mathrm{Ar}$ & $\mathrm{R}$ & Product 7, 8 & Yield 7, \% & Yield 8, \% & $\begin{array}{l}7: \mathbf{8} \\
\left(\text { in DMSO- } d_{6}\right)\end{array}$ \\
\hline $3 e$ & $4-\mathrm{MeOC}_{6} \mathrm{H}_{4}$ & $\mathrm{H}$ & $\mathbf{a}$ & 72 & 89 & $81: 19$ \\
\hline $3 f$ & $4-\mathrm{ClC}_{6} \mathrm{H}_{4}$ & $\mathrm{H}$ & b & 89 & 89 & $76: 24$ \\
\hline $3 \mathbf{h}$ & 2-Th & $\mathrm{H}$ & c & 86 & 92 & $93: 7$ \\
\hline $3 \mathbf{j}$ & $2-\mathrm{Th}$ & $\mathrm{Me}$ & d & 85 & 80 & $97: 3$ \\
\hline
\end{tabular}<smiles>CCOC(=O)c1cc(=O)c(C(=O)c2cccs2)co1</smiles>

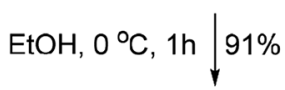<smiles></smiles>

12<smiles>O=C1CC2(O)C(=O)Nc3ncccc3N2C=C1C(=O)c1cccs1</smiles>

11
Scheme 5 Reaction of pyrone 1e with 2,3-diaminopyridine.

stable polycyclic pyridones 7 and $\mathbf{8}$ (Scheme 4 and Table 3). It was found that reflux of compounds 3 in $\mathrm{EtOH}$ without the addition of acid leads to the formation of dihydropyridones 7 in $72-89 \%$. Although quinoxalines 8 were detected in the ${ }^{1} \mathrm{H}$ NMR spectra of products 7 (the content reached $24 \%$ ), structures 7 are proved by the data of elemental analysis and the IR spectra of the obtained precipitates 7 exhibit the absence of the characteristic bands of the carbonyl groups of pyridones 8 at 1683$1710 \mathrm{~cm}^{-1}$. The obtained result probably shows that the formation of compounds 8 occurs upon the dissolution of dihydropyridone 7 in DMSO- $d_{6}$. Recrystallization of compounds 7 from acetic acid or boiling in EtOH with the addition of hydrochloric acid lead to the formation of dehydration products 8 in $80-92 \%$.

Enaminones 3a,d, which were obtained from aniline and $o$ aminophenol, undergo cyclization in the presence of acids gave thermodynamically more stable pyridones 9 in $78-79 \%$ (Scheme 4). On the other hand, it was important to find ways for the modification of such polycarbonyl ligands into structures containing heterocyclic substituents by reactions on the active diketobutanoic moiety. Using enaminone $3 \mathbf{c}$ as a representative example, it was shown that this compound is able to react with $o$-aminophenol $(\mathbf{2 b})$ under reflux in benzene to form benzoxazine 10 in $85 \%$ yield.

The reaction of thenoylpyrone 1e with 2,3-diaminopyridine (2j) currently led to dihydropyridone $\mathbf{1 1}$ (the content of dehydratation product 12 is $8 \%$ in DMSO- $d_{6}$ ) in $91 \%$ yield without the isolation of polycarbonyl intermediate 3 (Scheme 5). Compound 11 gave pyrido[1,2-a]quinoxaline-6,8-dione 12 in $81 \%$ yield under heating in $\mathrm{AcOH}$. The structure of products 11,12 was supported by the literature data ${ }^{23}$ regarding reactions of 2,3-diaminopyridones with polycarbonyl compounds.

\section{Conclusions}

In summary, we have found a convenient, convergent, and effective approach for the introduction of a triketone moiety into various amines using ethyl esters of 5-acyl-4-pyrone-2carboxylic acids, which allows the synthesis of a wide range of new polycarbonyl Schiff base ligands and salphen structures. Important advantages of this reaction include its wide substrate scope, catalyst free, atom economy, high yields, mild conditions and easy performing and isolation of products without the use of chromatography. Obtained polycarbonyl compounds in the solution of $\mathrm{CDCl}_{3}$ exist as several tautomers, the major tautomer has $5 E$-configuration of the double bond. The found strategy for the introduction of the triketone moiety can be used further for the deep functionalization of polymers and biopolymers containing a free amino group for the creation of novel catalysts or materials.

\section{Experimental}

\section{General}

NMR spectra were recorded on Bruker DRX-400 $\left({ }^{1} \mathrm{H}-400 \mathrm{MHz}\right.$ and $\left.{ }^{13} \mathrm{C}-100 \mathrm{MHz}\right)$ and Bruker Avance III-500 $\left({ }^{1} \mathrm{H}-500 \mathrm{MHz}\right.$ and ${ }^{13} \mathrm{C}-126 \mathrm{MHz}$ ) spectrometers in DMSO- $d_{6}$ or $\mathrm{CDCl}_{3}$. Chemical shifts are reported relative to TMS, $\mathrm{CHCl}_{3}(\delta=$ $7.26 \mathrm{ppm},{ }^{1} \mathrm{H}$ NMR), DMSO- $d_{6}\left(\delta=2.50 \mathrm{ppm},{ }^{1} \mathrm{H} \mathrm{NMR}\right), \mathrm{CDCl}_{3}$ $\left(\delta=77.06 \mathrm{ppm},{ }^{13} \mathrm{C} \mathrm{NMR}\right)$ and DMSO- $d_{6}\left(\delta=39.52 \mathrm{ppm},{ }^{13} \mathrm{C}\right.$ NMR) as internal standards. IR spectra were recorded on a Shimadzu IRSpirit-T (QATR-S) instrument (FTIR mode, ZnSe crystal). Elemental analysis was performed on a PerkinElmer PE 2400 automatic analyzer. The mass spectrum (ESI-MS) of compound 5 was measured with a Waters Xevo QTof instrument. All solvents used were dried and distilled per standard procedures. Melting points were determined on a Stuart SMP40 apparatus. Pyrones 1 were prepared according the literature procedure. $^{24}$

\section{General approach for the preparation of enaminodiones 3}

Pyrone 1 (1.11 mmol) was added to EtOH $(6 \mathrm{~mL})$ containing amine 2 (1.11 mmol) (for $3 \mathrm{~m}-2.22 \mathrm{mmol}$ ) under cooling on an 
ice bath. The resulted mixture was stirred at $0{ }^{\circ} \mathrm{C}$ for $1 \mathrm{~h}$ (for $3 \mathbf{k}-$ at $20{ }^{\circ} \mathrm{C}$ for $2 \mathrm{~h}$, or $3 \mathrm{~m}$ - at $20{ }^{\circ} \mathrm{C}$ for 1 day). The precipitate was filtered and washed with $\mathrm{EtOH}$ (for $3 \mathbf{i}$, the reaction mixture was additionally maintained at $-20{ }^{\circ} \mathrm{C}$ for $12 \mathrm{~h}$ ).

(2Z,5E)-Ethyl-5-benzoyl-2-hydroxy-4-oxo-6-(phenylamino) hexa-2,5-dienoate (3a). Yield 87\% (0.353 g), yellow powder, mp 128-129 ${ }^{\circ}$ C. IR (ATR): 2987, 1728, 1606, 1575, 1252, $847 \mathrm{~cm}^{-1}$. This compound exists in $\mathrm{CDCl}_{3}$ as a mixture of tautomers $E$ 3a : $Z$-3a : keto-3a $=82: 13: 5 .{ }^{1} \mathrm{H}$ NMR $\left(\mathrm{CDCl}_{3}, \mathrm{ppm}\right) \delta: E-3 \mathbf{a}$ $(82 \%)-1.31$ (t, $J=7.1 \mathrm{~Hz}, 3 \mathrm{H}, \mathrm{Me}), 4.30$ (q, $J=7.1 \mathrm{~Hz}, 2 \mathrm{H}$, $\left.\mathrm{OCH}_{2}\right), 7.01(\mathrm{~s}, 1 \mathrm{H},=\mathrm{CH}), 7.10(\mathrm{~d}, J=7.7 \mathrm{~Hz}, 2 \mathrm{H}, \mathrm{H}-2, \mathrm{H}-6 \mathrm{Ph})$, $7.22(\mathrm{tt}, J=7.4 \mathrm{~Hz}, J=1.1 \mathrm{~Hz}, 1 \mathrm{H}, \mathrm{H}-4 \mathrm{Ph}), 7.38(\mathrm{dd}, J=7.7 \mathrm{~Hz}, J$ $=7.4 \mathrm{~Hz}, 2 \mathrm{H}, \mathrm{H}-3, \mathrm{H}-5 \mathrm{Ph}), 7.48(\mathrm{dd}, J=7.8 \mathrm{~Hz}, J=7.2 \mathrm{~Hz}, 2 \mathrm{H}$, H-3, H-5 Ph') 7.57 (tt, $\left.J=7.8 \mathrm{~Hz}, J=1.2 \mathrm{~Hz}, 1 \mathrm{H}, \mathrm{H}-4 \mathrm{Ph}^{\prime}\right), 7.73$ (dd, $\left.J=7.2 \mathrm{~Hz}, J=1.4 \mathrm{~Hz}, 1 \mathrm{H}, \mathrm{H}-2, \mathrm{H}-6 \mathrm{Ph}^{\prime}\right), 8.08$ (d, $J=13.4 \mathrm{~Hz}$, $1 \mathrm{H},=\mathrm{CHN}), 12.41$ (d, $J=13.4 \mathrm{~Hz}, 1 \mathrm{H}, \mathrm{NH}), 14.36(\mathrm{~s}, 1 \mathrm{H}, \mathrm{OH})$; ${ }^{13} \mathrm{C} \mathrm{NMR}\left(\mathrm{CDCl}_{3}, \mathrm{ppm}\right) \delta 14.1,62.1,103.9,111.5,117.9,126.2$, 128.6, 129.3, 130.1, 132.2, 138.5, 139.8, 153.3, 160.1, 162.6, 194.08, 194.12. Anal. calcd for $\mathrm{C}_{21} \mathrm{H}_{19} \mathrm{NO}_{5}$ : C, 69.03; H, 5.24; N, 3.83. Found: C, 69.08; H, 5.13; N, 4.04.

(2Z,5E)-Ethyl-2-hydroxy-5-(4-methoxybenzoyl)-4-oxo-6(phenylamino)hexa-2,5-dienoate (3b). Yield 85\% (0.373 g), yellow powder, $\mathrm{mp} 131-133^{\circ} \mathrm{C}$. IR (ATR): 3205, 3005, 2849, 1715, $1580,1245,1108,1023 \mathrm{~cm}^{-1}$. This compound exists in $\mathrm{CDCl}_{3}$ as a mixture of tautomers $E-\mathbf{3 b}: Z-3 \mathbf{b}:$ keto-3b $=95: 2: 3 .{ }^{1} \mathrm{H}$ NMR $\left(\mathrm{CDCl}_{3}, \mathrm{ppm}\right) \delta: E-3 \mathbf{b}(95 \%)-1.31(\mathrm{t}, J=7.1 \mathrm{~Hz}, 3 \mathrm{H}, \mathrm{Me})$, 3.89 (s, 3H, MeO), 4.29 (q, $\left.J=7.1 \mathrm{~Hz}, 2 \mathrm{H}, \mathrm{OCH}_{2}\right), 6.90(\mathrm{~s}, 1 \mathrm{H},=$ $\mathrm{CH}), 6.97$ (d, $\left.\mathrm{AA}^{\prime} \mathrm{BB}^{\prime}, J=8.9 \mathrm{~Hz}, 2 \mathrm{H}, \mathrm{H}-3 \mathrm{H}-5 \mathrm{Ar}\right), 7.12$ (d, $J=$ $7.7 \mathrm{~Hz}, 2 \mathrm{H}, \mathrm{H}-2, \mathrm{H}-6 \mathrm{Ph}$ ), 7.21 (tt, $J=7.4 \mathrm{~Hz}, J=0.9 \mathrm{~Hz}, 1 \mathrm{H}, \mathrm{H}-4$ $\mathrm{Ph}$ ), 7.39 (dd, $J=7.9 \mathrm{~Hz}, J=7.4 \mathrm{~Hz}, 2 \mathrm{H}, \mathrm{H}-3, \mathrm{H}-5 \mathrm{Ph}$ ), 7.74 (d, $\left.\mathrm{AA}^{\prime} \mathrm{BB}^{\prime}, J=8.9 \mathrm{~Hz}, 2 \mathrm{H}, \mathrm{H}-2, \mathrm{H}-6 \mathrm{Ar}\right), 8.05(\mathrm{~d}, J=13.3 \mathrm{~Hz}, 1 \mathrm{H},=$ $\mathrm{CHN}$ ), 12.34 (d, $J=13.3 \mathrm{~Hz}, 1 \mathrm{H}, \mathrm{NH}), 14.38$ (s, $1 \mathrm{H}, \mathrm{OH}) ;{ }^{13} \mathrm{C}$ NMR $\left(\mathrm{CDCl}_{3}, \mathrm{ppm}\right) \delta 14.0(\mathrm{Me}), 55.5(\mathrm{MeO}), 62.1\left(\mathrm{CH}_{2}\right), 103.9(\mathrm{C}-$ 3), 111.7 (C-5), 113.8 (C-3, C-5 Ar), 117.8 (C-2, C-6 Ph), 126.0 (C-4 Ph), 130.0 (C-3, C-5 Ph), 131.8 (C-2, C-6 Ar), 132.1 (C-1 Ar), 138.6 (C-1 Ph), 152.4 (C-6), 159.9 (C-2), 162.6 (C-1), 163.1 (C-4 Ar), $193.0($ Ar-C-4' $=\mathrm{O}), 193.9(\mathrm{C}-4=\mathrm{O})$. Anal. calcd for $\mathrm{C}_{22} \mathrm{H}_{21} \mathrm{NO}_{6}: \mathrm{C}$, 66.83; H, 5.35; N, 3.54. Found: C, 66.61; H, 5.27; N, 3.86.

(2Z,5E)-Ethyl-5-benzoyl-2-hydroxy-6-((2-hydroxyphenyl) amino)-4-oxohexa-2,5-dienoate (3c). Yield 68\% (0.288 g), yellow powder, mp $172-174{ }^{\circ} \mathrm{C}$. IR (ATR): $3057,1733,1640,1608,1562$, 1446, 1251, 1157, 1099, $847 \mathrm{~cm}^{-1}$. This compound exists in $\mathrm{CDCl}_{3}$ as a mixture of tautomers $E-3 \mathbf{c}: Z-3 \mathbf{c}:$ keto-3c $=$ $76: 17: 7 .{ }^{1} \mathrm{H} \mathrm{NMR}\left(\mathrm{CDCl}_{3}, \mathrm{ppm}\right) \delta: E-3 \mathrm{c}(76 \%)-1.31(\mathrm{t}, J=$ $7.1 \mathrm{~Hz}, 3 \mathrm{H}, \mathrm{Me}$ ), 4.30 (q, $\left.J=7.1 \mathrm{~Hz}, 2 \mathrm{H}, \mathrm{OCH}_{2}\right), 6.79(\mathrm{br}, 1 \mathrm{H}$, HOAr), 6.86-6.93 (m, 1H, Ar), 6.95-7.10 (m, 4H, Ar, =CH), 7.48 (t, $J=7.4 \mathrm{~Hz}, 2 \mathrm{H}, \mathrm{H}-3, \mathrm{H}-5 \mathrm{Ph}), 7.57$ (t, $J=7.2 \mathrm{~Hz}, 1 \mathrm{H}, \mathrm{H}-4 \mathrm{Ph}$ ), $7.73(\mathrm{~d}, J=7.3 \mathrm{~Hz}, 2 \mathrm{H}, \mathrm{H}-2, \mathrm{H}-6 \mathrm{Ph}), 8.13$ (d, $J=13.3 \mathrm{~Hz}, 1 \mathrm{H},=$ CHN), 12.60 (d, $J=13.3 \mathrm{~Hz}, 1 \mathrm{H}, \mathrm{NH}), 14.80$ (s, 1H, OH). Anal. calcd for $\mathrm{C}_{21} \mathrm{H}_{19} \mathrm{NO}_{6}$ : C, 66.14; H, 5.02; N, 3.67. Found: C, 66.32; $\mathrm{H}, 4.91 ; \mathrm{N}, 3.38$.

(2Z,5E)-Ethyl-5-(4-chlorobenzoyl)-2-hydroxy-6-(2hydroxyphenylamino)-4-oxohexa-2,5-dienoate (3d). Yield $81 \%$ (0.374 g), yellow powder, mp 180-182 ${ }^{\circ} \mathrm{C}$. IR (ATR): 3190,1734 , $1609,1576,1248,744 \mathrm{~cm}^{-1}$. This compound exists in $\mathrm{CDCl}_{3}$ as a mixture of tautomers $E$-3d : $Z$-3d : keto-3d $=76: 17: 7 .{ }^{1} \mathrm{H}$ $\mathrm{NMR}\left(\mathrm{CDCl}_{3}, \mathrm{ppm}\right) \delta: E-3 d(76 \%)-1.31(\mathrm{t}, J=7.1 \mathrm{~Hz}, 3 \mathrm{H}, \mathrm{Me})$, $4.30\left(\mathrm{q}, J=7.1 \mathrm{~Hz}, 2 \mathrm{H}, \mathrm{OCH}_{2}\right), 6.10(\mathrm{br}, 1 \mathrm{H}, \mathrm{HOAr}), 6.88-7.12(\mathrm{~m}$, $5 \mathrm{H}, \mathrm{Ar},=\mathrm{CH}), 7.45\left(\mathrm{~d}, \mathrm{AA}^{\prime} \mathrm{BB}^{\prime}, J=8.4 \mathrm{~Hz}, 2 \mathrm{H}, \mathrm{H}-3, \mathrm{H}-5 \mathrm{Ar}^{\prime}\right), 7.68$ $\left(\mathrm{d}, \mathrm{AA}^{\prime} \mathrm{BB}^{\prime}, J=8.4 \mathrm{~Hz}, 2 \mathrm{H}, \mathrm{H}-2, \mathrm{H}-6 \mathrm{Ar}^{\prime}\right), 8.10$ (d, $J=13.6 \mathrm{~Hz}, 1 \mathrm{H}$, $=\mathrm{CHN}), 12.59(\mathrm{~d}, J=13.6 \mathrm{~Hz}, 1 \mathrm{H}, \mathrm{NH}), 14.49$ (s, 1H, OH). Anal. calcd for $\mathrm{C}_{21} \mathrm{H}_{18} \mathrm{ClNO}_{6}: \mathrm{C}, 60.66 ; \mathrm{H}, 4.36 ; \mathrm{N}, 3.37$. Found: $\mathrm{C}$, $60.61 ; \mathrm{H}, 4.10 ; \mathrm{N}, 3.30$.

(2Z,5E)-Ethyl-6-(2-aminophenylamino)-2-hydroxy-5-(4methoxybenzoyl)-4-oxohexa-2,5-dienoate (3e). Yield 83\% (0.378 g), red powder, $\mathrm{mp} 131-133{ }^{\circ} \mathrm{C}$. IR (ATR): 3394, 1731, 1611, 1595, $1246,749 \mathrm{~cm}^{-1}$. This compound exists in $\mathrm{CDCl}_{3}$ as a mixture of tautomers $E$-3e : $Z$-3e : keto-3e $=79: 15: 6 .{ }^{1} \mathrm{H}$ NMR $\left(\mathrm{CDCl}_{3}\right.$, ppm) $\delta: E-3 \mathrm{e}(79 \%)-1.31(\mathrm{t}, J=7.1 \mathrm{~Hz}, 3 \mathrm{H}, \mathrm{Me}), 3.77(\mathrm{br}, 2 \mathrm{H}$, $\mathrm{NH}_{2}$ ), 3.88 (s, 3H, MeO), 4.29 (q, $J=7.1 \mathrm{~Hz}, 2 \mathrm{H}, \mathrm{OCH}_{2}$ ), 6.78$6.87(\mathrm{~m}, 2 \mathrm{H}, \mathrm{Ar}), 6.92-7.00\left(\mathrm{~m}, 4 \mathrm{H},=\mathrm{CH}, \mathrm{Ar}, \mathrm{Ar}^{\prime}\right), 7.07(\mathrm{td}, J=$ $7.6 \mathrm{~Hz}, J=0.9 \mathrm{~Hz}, 1 \mathrm{H}, \mathrm{H}-4 \mathrm{Ar}), 7.74\left(\mathrm{~d}, \mathrm{AA}^{\prime} \mathrm{BB}^{\prime}, J=8.7 \mathrm{~Hz}, 2 \mathrm{H}, \mathrm{H}-\right.$ 2, H-6 Ar'), 7.95 (d, $J=13.1 \mathrm{~Hz}, 1 \mathrm{H},=\mathrm{CHN}), 12.24(\mathrm{~d}, J=$ $13.1 \mathrm{~Hz}, 1 \mathrm{H}, \mathrm{NH}), 14.33(\mathrm{~s}, 1 \mathrm{H}, \mathrm{OH})$. Anal. calcd for $\mathrm{C}_{22} \mathrm{H}_{22} \mathrm{~N}_{2} \mathrm{O}_{6}$ : C, 64.38; H, 5.40; N, 6.83. Found: C, 64.59; H, 5.60; N, 6.64.

(2Z,5E)-Ethyl-6-(2-aminophenylamino)-5-(4-chlorobenzoyl)2-hydroxy-4-oxohexa-2,5-dienoate (3f). Yield 78\% (0.359 g), orange powder, $\mathrm{mp} 138-140{ }^{\circ} \mathrm{C}$. IR (ATR): 3404, 3351, 3068, $1723,1597,117,841 \mathrm{~cm}^{-1}$. This compound exists in $\mathrm{CDCl}_{3}$ as a mixture of tautomers $E-3 f: Z-3 f:$ keto-3f $=78: 16: 6 .{ }^{1} \mathrm{H} \mathrm{NMR}$ $\left(\mathrm{CDCl}_{3}, \mathrm{ppm}\right) \delta: E-3 f(78 \%)-1.34(\mathrm{t}, J=7.1 \mathrm{~Hz}, 3 \mathrm{H}, \mathrm{Me}), 3.79(\mathrm{br}$, $\left.2 \mathrm{H}, \mathrm{NH}_{2}\right), 4.32\left(\mathrm{q}, J=7.1 \mathrm{~Hz}, 2 \mathrm{H}, \mathrm{OCH}_{2}\right), 6.81-6.90(\mathrm{~m}, 2 \mathrm{H}, \mathrm{Ar})$, $6.98(\mathrm{~d}, J=7.9 \mathrm{~Hz}, 1 \mathrm{H}, \mathrm{H}-6 \mathrm{Ar}), 7.00(\mathrm{~s}, 1 \mathrm{H},=\mathrm{CH}), 7.07(\mathrm{td}, J=$ $7.7 \mathrm{~Hz}, J=0.9 \mathrm{~Hz}, 1 \mathrm{H}, \mathrm{H}-4 \mathrm{Ar}$ ), 7.47 (d, $\mathrm{AA}^{\prime} \mathrm{BB}^{\prime}, J=8.4 \mathrm{~Hz}, 2 \mathrm{H}, \mathrm{H}-$ 3, H-5 $\mathrm{Ar}^{\prime}$ ), 7.69 (d, $\left.\mathrm{AA}^{\prime} \mathrm{BB}^{\prime}, J=8.4 \mathrm{~Hz}, 2 \mathrm{H}, \mathrm{H}-2, \mathrm{H}-6 \mathrm{Ar}^{\prime}\right), 7.97$ (d, $J$ $=13.2 \mathrm{~Hz}, 1 \mathrm{H},=\mathrm{CHN}), 12.33(\mathrm{~d}, J=13.2 \mathrm{~Hz}, 1 \mathrm{H}, \mathrm{NH}), 14.28(\mathrm{~s}$, $1 \mathrm{H}, \mathrm{OH})$. Anal. calcd for $\mathrm{C}_{21} \mathrm{H}_{19} \mathrm{ClN}_{2} \mathrm{O}_{5}: \mathrm{C}, 60.80 ; \mathrm{H}, 4.62 ; \mathrm{N}$, 6.72. Found: C, 60.78; H, 4.62; N, 6.67.

(2Z,5E)-Ethyl-5-(2-naphthoyl)-6-(2-aminophenylamino)-2hydroxy-4-oxohexa-2,5-dienoate (3g). Yield $90 \%\left(\begin{array}{llll}0.430 & \mathrm{~g}\end{array}\right)$, orange powder, mp 169-171 ${ }^{\circ} \mathrm{C}$. IR (ATR): 3401, 3183, 2987, $1728,1532,1246,1101,827 \mathrm{~cm}^{-1}$. This compound exists in $\mathrm{CDCl}_{3}$ as a mixture of tautomers $E-\mathbf{3 g}: Z-3 \mathrm{~g}:$ keto-3g $=$ $79: 13: 8 .{ }^{1} \mathrm{H} \mathrm{NMR}\left(\mathrm{CDCl}_{3}, \mathrm{ppm}\right) \delta: E-3 \mathrm{~g}(79 \%)-1.25(\mathrm{t}, J=$ $7.1 \mathrm{~Hz}, 3 \mathrm{H}, \mathrm{OCH}_{2} \mathrm{Me}$ ), $3.79\left(\mathrm{br}, 2 \mathrm{H}, \mathrm{NH}_{2}\right), 4.27$ (q, $J=7.1 \mathrm{~Hz}, 2 \mathrm{H}$, $\left.\mathrm{OCH}_{2}\right), 6.79(\mathrm{td}, J=7.4 \mathrm{~Hz}, J=0.9 \mathrm{~Hz}, 1 \mathrm{H}, \mathrm{H}-5 \mathrm{Ar}), 6.85(\mathrm{dd}, J=$ $8.0 \mathrm{~Hz}, J=0.9 \mathrm{~Hz}, 1 \mathrm{H}, \mathrm{H}-3 \mathrm{Ar}$ ), 6.95 (d, $J=7.5 \mathrm{~Hz}, 1 \mathrm{H}, \mathrm{H}-6 \mathrm{Ar}$ ), $7.08(\mathrm{td}, J=7.7 \mathrm{~Hz}, J=0.9 \mathrm{~Hz}, 1 \mathrm{H}, \mathrm{H}-4 \mathrm{Ar}), 7.10(\mathrm{~s}, 1 \mathrm{H},=\mathrm{CH})$, 7.51-7.68 (m, 2H, Naph), 7.84 (dd, $J=8.5 \mathrm{~Hz}, J=1.5 \mathrm{~Hz}, 1 \mathrm{H}$, Naph), 7.89-8.00 (m, 3H, Naph), 8.07 (d, $J=13.1 \mathrm{~Hz}, 1 \mathrm{H},=$ CHN), 8.24 (d, $J=0.7 \mathrm{~Hz}, 1 \mathrm{H}, \mathrm{H}-1 \mathrm{Naph}), 12.38$ (d, $J=13.1 \mathrm{~Hz}$, $1 \mathrm{H}, \mathrm{NH}), 14.34(\mathrm{~s}, 1 \mathrm{H}, \mathrm{OH})$. Anal. calcd for $\mathrm{C}_{25} \mathrm{H}_{22} \mathrm{~N}_{2} \mathrm{O}_{5}$ : C, 69.76; H, 5.15; N, 6.51. Found: C, 69.88; H, 5.14; N, 6.30.

(2Z,5E)-Ethyl-6-(2-aminophenylamino)-2-hydroxy-4-oxo-5(thiophene-2-carbonyl)hexa-2,5-dienoate (3h). Yield 90\% (0.386 g), orange powder, mp $143-145{ }^{\circ} \mathrm{C}$. IR (ATR): 3416, 3345, 3135, $1722,1635,1578,1248,836 \mathrm{~cm}^{-1}$. This compound exists in $\mathrm{CDCl}_{3}$ as a mixture of tautomers $E-3 \mathbf{h}: Z-3 \mathbf{h}:$ keto-3h $=$ $89: 8: 3 .{ }^{1} \mathrm{H}$ NMR $\left(\mathrm{CDCl}_{3}, \mathrm{ppm}\right) \delta: E-3 \mathrm{~h}(89 \%)-1.33(\mathrm{t}, J=$ $7.1 \mathrm{~Hz}, 3 \mathrm{H}, \mathrm{Me}), 3.79\left(\mathrm{br}, 2 \mathrm{H}, \mathrm{NH}_{2}\right), 4.31$ (q, $J=7.1 \mathrm{~Hz}, 2 \mathrm{H}$, $\left.\mathrm{OCH}_{2}\right), 6.82-6.88(\mathrm{~m}, 2 \mathrm{H}, \mathrm{H}-5, \mathrm{H}-3 \mathrm{Ar}), 7.04(\mathrm{dd}, J=7.2 \mathrm{~Hz}, J=$ $1.0 \mathrm{~Hz}, 1 \mathrm{H}, \mathrm{H}-6 \mathrm{Ar}), 7.04(\mathrm{~s}, 1 \mathrm{H},=\mathrm{CH}), 7.09(\mathrm{td}, J=7.9 \mathrm{~Hz}, J=$ $1.1 \mathrm{~Hz}, 1 \mathrm{H}, \mathrm{H}-4 \mathrm{Ar}$ ), 7.14 (dd, $J=5.0 \mathrm{~Hz}, J=3.8 \mathrm{~Hz}, 1 \mathrm{H}, \mathrm{H}-4 \mathrm{Th}$ ), $7.55(\mathrm{dd}, J=3.8 \mathrm{~Hz}, J=1.0 \mathrm{~Hz}, 1 \mathrm{H}, \mathrm{H}-3 \mathrm{Th}), 7.67$ (dd, $J=5.0 \mathrm{~Hz}, J$ 
$=1.0 \mathrm{~Hz}, 1 \mathrm{H}, \mathrm{H}-5 \mathrm{Th}), 8.14(\mathrm{~d}, J=13.1 \mathrm{~Hz}, 1 \mathrm{H},=\mathrm{CHN}), 12.22(\mathrm{~d}$, $J=13.1 \mathrm{~Hz}, 1 \mathrm{H}, \mathrm{NH}), 14.28(\mathrm{~s}, 1 \mathrm{H}, \mathrm{OH})$. Anal. calcd for $\mathrm{C}_{19} \mathrm{H}_{18} \mathrm{~N}_{2} \mathrm{O}_{5} \mathrm{~S}: \mathrm{C}, 59.06 ; \mathrm{H}, 4.70 ; \mathrm{N}, 7.25$. Found: C, 58.88; H, 4.88 ; N, 7.49.

(2Z,5E)-Ethyl-5-((2-aminophenylamino)methylene)-2hydroxy-7,7-dimethyl-4,6-dioxooct-2-enoate (3i). Yield $61 \%$ (0.244 g), yellow powder, mp 104-106 ${ }^{\circ} \mathrm{C}$. IR (ATR): 3223, 3100, 2959, 2921, 1695, 1677, 1637, 1504, 1108, $882 \mathrm{~cm}^{-1}$. This compound exists in $\mathrm{CDCl}_{3}$ as a mixture of tautomers $E$-3i : $Z$ $3 \mathbf{i}:$ keto-3i $=79: 14: 7 .{ }^{1} \mathrm{H}$ NMR $\left(\mathrm{CDCl}_{3}, \mathrm{ppm}\right) \delta: E-3 \mathbf{i}(79 \%)-$ 1.35 (s, 9H, $t$-Bu), 1.38 (t, $J=7.1 \mathrm{~Hz}, 3 \mathrm{H}, \mathrm{Me}), 3.75\left(\mathrm{br}, 2 \mathrm{H}, \mathrm{NH}_{2}\right.$ ), 4.36 (q, $\left.J=7.1 \mathrm{~Hz}, 2 \mathrm{H}, \mathrm{OCH}_{2}\right), 6.59(\mathrm{~s}, 1 \mathrm{H},=\mathrm{CH}), 6.83-6.92(\mathrm{~m}$, $2 \mathrm{H}, \mathrm{Ar}), 7.05(\mathrm{dd}, J=7.8 \mathrm{~Hz}, J=0.9 \mathrm{~Hz}, 1 \mathrm{H}, \mathrm{H}-6 \mathrm{Ar}), 7.11(\mathrm{td}, J=$ $7.6 \mathrm{~Hz}, J=1.1 \mathrm{~Hz}, 1 \mathrm{H}, \mathrm{H}-4 \mathrm{Ar}), 7.86(\mathrm{~d}, J=12.4 \mathrm{~Hz}, 1 \mathrm{H},=\mathrm{CHN})$, $11.95(\mathrm{~d}, J=12.4 \mathrm{~Hz}, 1 \mathrm{H}, \mathrm{NH}), 14.36(\mathrm{~s}, 1 \mathrm{H}, \mathrm{OH})$. Anal. calcd for $\mathrm{C}_{19} \mathrm{H}_{24} \mathrm{~N}_{2} \mathrm{O}_{5}$ : C, 63.22; H, 6.71; N, 7.77. Found: C, 63.53; H, $6.58 ; \mathrm{N}, 7.89$.

(2Z,5E)-Ethyl-6-(2-amino-4,5-dimethylphenylamino)-2hydroxy-4-oxo-5-(thiophene-2-carbonyl)hexa-2,5-dienoate Yield 96\% (0.442 g), red powder, mp 157-159 ${ }^{\circ} \mathrm{C}$. IR (ATR): 3411 , $3370,2992,1717,1590,1256,794 \mathrm{~cm}^{-1}$. This compound exists in $\mathrm{CDCl}_{3}$ as a mixture of tautomers $E-3 \mathbf{j}: Z-3 \mathbf{j}:$ keto-3j $=$ $87: 9: 4 .{ }^{1} \mathrm{H}$ NMR $\left(\mathrm{CDCl}_{3}, \mathrm{ppm}\right) \delta: E-3 \mathbf{j}(87 \%)-1.33(\mathrm{t}, J=$ $7.1 \mathrm{~Hz}, 3 \mathrm{H}, \mathrm{OCH}_{2} \mathrm{Me}$ ), 2.16 (s, 3H, Me), 2.19 (s, 3H, Me), 3.59 (br, $\left.2 \mathrm{H}, \mathrm{NH}_{2}\right), 4.31\left(\mathrm{q}, J=7.1 \mathrm{~Hz}, 2 \mathrm{H}, \mathrm{OCH}_{2}\right), 6.65(\mathrm{~s}, 1 \mathrm{H}, \mathrm{Ar}), 6.80(\mathrm{~s}$, $1 \mathrm{H}, \mathrm{Ar}), 7.02(\mathrm{~s}, 1 \mathrm{H},=\mathrm{CH}), 7.14(\mathrm{dd}, 1 \mathrm{H}, J=5.0 \mathrm{~Hz}, J=3.8 \mathrm{~Hz}$, H-4 Th), 7.54 (dd, $1 \mathrm{H}, J=3.8 \mathrm{~Hz}, J=1.1 \mathrm{~Hz}, \mathrm{H}-3 \mathrm{Th}$ ), 7.67 (dd, $1 \mathrm{H}, J=5.0 \mathrm{~Hz}, J=1.1 \mathrm{~Hz}, \mathrm{H}-5 \mathrm{Th}), 8.11(\mathrm{~d}, J=13.2 \mathrm{~Hz}, 1 \mathrm{H},=$ $\mathrm{CHN}$ ), 12.23 (d, $J=13.2 \mathrm{~Hz}, 1 \mathrm{H}, \mathrm{NH}), 14.32$ (s, $1 \mathrm{H}, \mathrm{OH}) ;{ }^{13} \mathrm{C}$ $\mathrm{NMR}\left(\mathrm{CDCl}_{3}, \mathrm{ppm}\right) \delta 14.1,19.0,19.4,62.1,103.5,111.6,119.5$, $120.1,124.8,127.8,128.7,132.9$, 133.3, 135.7, 136.3, 145.3, 153.9, 159.7, 162.7, 185.3, 193.0. Anal. calcd for $\mathrm{C}_{21} \mathrm{H}_{22} \mathrm{~N}_{2} \mathrm{O}_{5} \mathrm{~S}: \mathrm{C}$, 60.85 ; H, 5.35; N, 6.76. Found: C, 60.51; H, 5.65; N, 6.65.

(2Z,5E)-Ethyl-5-benzoyl-2-hydroxy-4-oxo-6-(pyridin-2-

ylamino)hexa-2,5-dienoate (3k). Yield 74\% (0.301 g), yellow powder, mp 159-160 ${ }^{\circ} \mathrm{C}$. IR (ATR): 3219, 2995, 1741, 1622, 1590, 1563, 1253, $887 \mathrm{~cm}^{-1}$. This compound exists in $\mathrm{CDCl}_{3}$ as a mixture of tautomers $E$-3k: $Z$-3k : keto-3k $=80: 13: 7 .{ }^{1} \mathrm{H}$ NMR $\left(\mathrm{CDCl}_{3}, \mathrm{ppm}\right) \delta$ : $E$-3k (80\%) - 1.35 (t, $\left.J=7.1 \mathrm{~Hz}, 3 \mathrm{H}, \mathrm{Me}\right)$, $4.34\left(\mathrm{q}, J=7.1 \mathrm{~Hz}, 2 \mathrm{H}, \mathrm{OCH}_{2}\right), 7.00(\mathrm{~d}, J=8.1 \mathrm{~Hz}, 1 \mathrm{H}, \mathrm{Py}), 7.10$ $(\mathrm{dd}, J=7.3 \mathrm{~Hz}, J=4.8 \mathrm{~Hz}, 1 \mathrm{H}, \mathrm{Py}), 7.12(\mathrm{~s}, 1 \mathrm{H},=\mathrm{CH}), 7.51(\mathrm{dd}, J$ $=7.7 \mathrm{~Hz}, J=7.3 \mathrm{~Hz}, 2 \mathrm{H}, \mathrm{H}-3, \mathrm{H}-5 \mathrm{Ph}), 7.61(\mathrm{tt}, J=7.7 \mathrm{~Hz}, J=$ $1.4 \mathrm{~Hz}, 1 \mathrm{H}, \mathrm{H}-4 \mathrm{Ph}), 7.73(\mathrm{td}, J=7.8 \mathrm{~Hz}, J=1.3 \mathrm{~Hz}, 1 \mathrm{H}, \mathrm{Py}), 7.79$ $(2 \mathrm{H}, \mathrm{dd}, J=7.9 \mathrm{~Hz}, J=1.4 \mathrm{~Hz}, \mathrm{H}-2, \mathrm{H}-6 \mathrm{Ph}), 8.30(\mathrm{dd}, J=4.8 \mathrm{~Hz}$, $J=1.3 \mathrm{~Hz}, 1 \mathrm{H}, \mathrm{Py}), 8.81(\mathrm{~d}, J=12.5 \mathrm{~Hz}, 1 \mathrm{H},=\mathrm{CHN}), 12.41(\mathrm{~d}, J$ $=12.5 \mathrm{~Hz}, 1 \mathrm{H}, \mathrm{NH}), 14.44(\mathrm{~s}, 1 \mathrm{H}, \mathrm{OH}) ;{ }^{13} \mathrm{C} \mathrm{NMR}\left(\mathrm{CDCl}_{3}, \mathrm{ppm}\right)$ $\delta 14.1,62.2,103.8,112.3,112.9,120.7,128.6,129.6,132.3,138.8$, 139.4, 149.0, 149.9, 151.2, 160.8, 162.5, 194.4, 194.8. Anal. calcd for $\mathrm{C}_{20} \mathrm{H}_{18} \mathrm{~N}_{2} \mathrm{O}_{5}$ : C, 65.57; $\mathrm{H}, 4.95 ; \mathrm{N}, 7.65$. Found: $\mathrm{C}, 65.62 ; \mathrm{H}$, 4.76 ; N, 7.94 .

(2Z,5E)-Ethyl-6-(1,5-dimethyl-3-oxo-2-phenyl-2,3-dihydro-1Hpyrazol-4-ylamino)-2-hydroxy-4-oxo-5-(thiophene-2-carbonyl)

hexa-2,5-dienoate (31). Yield 83\% (0.444 g), yellow powder, $\mathrm{mp}$ 170-172 ${ }^{\circ}$ C. IR (ATR): 3101, 1715, 1668, 1587, 1493, 1246, 1020, $910,846 \mathrm{~cm}^{-1}$. This compound exists in $\mathrm{CDCl}_{3}$ as a mixture of tautomers $E$-3l : $Z$-3l : keto-3l = $92: 4: 4 .{ }^{1} \mathrm{H} \mathrm{NMR}\left(\mathrm{CDCl}_{3}, \mathrm{ppm}\right)$ $\delta: E-31(92 \%)-1.34\left(\mathrm{t}, J=7.1 \mathrm{~Hz}, 3 \mathrm{H}, \mathrm{OCH}_{2} \mathrm{Me}\right), 2.38$ (s, 3H, Me), $3.11(\mathrm{~s}, 3 \mathrm{H}, \mathrm{Me}), 4.32\left(\mathrm{q}, J=7.1 \mathrm{~Hz}, 2 \mathrm{H}, \mathrm{OCH}_{2}\right), 7.12(\mathrm{dd}, J=$ $5.0 \mathrm{~Hz}, J=3.9 \mathrm{~Hz}, 1 \mathrm{H}, \mathrm{H}-4 \mathrm{Th}), 7.15(\mathrm{~s}, 1 \mathrm{H},=\mathrm{CH}), 7.33-7.38(\mathrm{~m}$, $3 \mathrm{H}, \mathrm{Ph}$ ), 7.48 (dd, $J=8.0 \mathrm{~Hz}, J=7.4 \mathrm{~Hz}, 2 \mathrm{H}, \mathrm{H}-3, \mathrm{H}-5 \mathrm{Ph}$ ), 7.60 $(\mathrm{dd}, J=5.0 \mathrm{~Hz}, J=0.8 \mathrm{~Hz}, 1 \mathrm{H}, \mathrm{H}-5 \mathrm{Th}), 7.64(\mathrm{dd}, J=3.9 \mathrm{~Hz}, J=$ $0.8 \mathrm{~Hz}, 1 \mathrm{H}, \mathrm{H}-3, \mathrm{Th}), 9.03$ (d, $J=12.6 \mathrm{~Hz}, 1 \mathrm{H},=\mathrm{CHN}$ ), 12.52 (d, $J$ $=12.6 \mathrm{~Hz}, 1 \mathrm{H}, \mathrm{NH}), 14.27(\mathrm{~s}, 1 \mathrm{H}, \mathrm{OH})$. Anal. calcd for $\mathrm{C}_{24} \mathrm{H}_{23} \mathrm{~N}_{3} \mathrm{O}_{6} \mathrm{~S}: \mathrm{C}, 59.86 ; \mathrm{H}, 4.81 ; \mathrm{N}$, 8.73. Found: C, 59.94; $\mathrm{H}$, $4.70 ; \mathrm{N}, 8.72$.

(2Z,5E)-Ethyl-2-hydroxy-4-oxo-6-(pyrimidin-2-ylamino)-5(thiophene-2-carbonyl)hexa-2,5-dienoate (3m). Yield 47\% (0.195 g), light yellow powder, mp 204-206 ${ }^{\circ} \mathrm{C}$. IR (ATR): 3272, 3096, 3083, 1736, 1616, 1595, 1562, 1434, 1248, 987, $843 \mathrm{~cm}^{-1}$. This compound exists in $\mathrm{CDCl}_{3}$ as a mixture of tautomers $E-3 \mathbf{m}: Z$ $3 \mathbf{m}:$ keto-3m = $93: 2: 5 .{ }^{1} \mathrm{H}$ NMR $\left(\mathrm{CDCl}_{3}, \mathrm{ppm}\right) \delta: E-3 \mathbf{m}(93 \%)-$ $1.35(\mathrm{t}, 3 \mathrm{H}, J=7.1 \mathrm{~Hz}, \mathrm{Me}), 4.34$ (q, $\left.J=7.1 \mathrm{~Hz}, 2 \mathrm{H}, \mathrm{OCH}_{2}\right), 6.99$ $(\mathrm{s}, 1 \mathrm{H},=\mathrm{CH}), 7.07(\mathrm{t}, J=4.8 \mathrm{~Hz}, 1 \mathrm{H}, \mathrm{H}-5$ pyrimidine), $7.18(\mathrm{dd}, J$ $=4.9 \mathrm{~Hz}, J=3.8 \mathrm{~Hz}, 1 \mathrm{H}, \mathrm{H}-4 \mathrm{Th}), 7.60(\mathrm{dd}, J=3.8 \mathrm{~Hz}, J=0.8 \mathrm{~Hz}$, 1H, H-3 Th), 7.73 (dd, $J=4.9 \mathrm{~Hz}, J=0.8 \mathrm{~Hz}, 1 \mathrm{H}, \mathrm{H}-5 \mathrm{Th}$ ), 8.57 (d, $J=4.8 \mathrm{~Hz}, 2 \mathrm{H}, \mathrm{H}-4, \mathrm{H}-6$ pyrimidine), $8.88(\mathrm{~d}, J=12.6 \mathrm{~Hz}, 1 \mathrm{H},=$ $\mathrm{CHN}$ ), 12.07 (d, $J=12.6 \mathrm{~Hz}, 1 \mathrm{H}, \mathrm{NH}), 14.50$ (s, 1H, OH). Anal. calcd for $\mathrm{C}_{17} \mathrm{H}_{15} \mathrm{~N}_{3} \mathrm{O}_{5} \mathrm{~S}: \mathrm{C}, 54.68 ; \mathrm{H}, 4.05 ; \mathrm{N}, 11.25$. Found: $\mathrm{C}$, 54.79; H, 4.06; N, 11.04 .

(2Z,5E)-Ethyl-5-((benzylamino)methylene)-2-hydroxy-7,7dimethyl-4,6-dioxooct-2-enoate (3n). Yield 54\% (0.215 g), yellow powder, mp 120-122 ${ }^{\circ} \mathrm{C}$. IR (ATR): 3281, 2967, 1730, 1622, 1595, 1249, 1172, $862 \mathrm{~cm}^{-1}$. This compound exists in $\mathrm{CDCl}_{3}$ as a mixture of tautomers $E-3 \mathbf{n}: Z-3 \mathbf{n}:$ keto-3n $=79: 11: 10 .{ }^{1} \mathrm{H}$ $\operatorname{NMR}\left(\mathrm{CDCl}_{3}, \mathrm{ppm}\right) \delta: E-3 \mathrm{~m}(79 \%)-1.20(\mathrm{~s}, 9 \mathrm{H}, t-\mathrm{Bu}), 1.34(\mathrm{t}, J=$ $7.1 \mathrm{~Hz}, 3 \mathrm{H}, \mathrm{Me}$ ), 4.32 (q, $\left.J=7.1 \mathrm{~Hz}, 2 \mathrm{H}, \mathrm{OCH}_{2}\right), 4.54$ (d, $J=$ $\left.6.0 \mathrm{~Hz}, 2 \mathrm{H}, \mathrm{CH}_{2} \mathrm{Ph}\right), 6.63(\mathrm{~s}, 1 \mathrm{H},=\mathrm{CH}), 7.28(\mathrm{~d}, J=7.1 \mathrm{~Hz}, 2 \mathrm{H}$, H-2, H-6 Ph), 7.32-7.44 (3H, m, Ph), 7.69 (d, $J=13.3 \mathrm{~Hz}, 1 \mathrm{H},=$ CHN), 10.67 (dt, $J=13.3 \mathrm{~Hz}, J=6.0 \mathrm{~Hz}, 1 \mathrm{H}, \mathrm{NH}), 14.43$ (s, 1H, $\mathrm{OH}$ ). Anal. calcd for $\mathrm{C}_{20} \mathrm{H}_{25} \mathrm{NO}_{5}$ : C, 66.84; H, 7.01; N, 3.90. Found: C, 67.00; H, 6.91; N, 4.11.

$(2 Z, 5 E)$-Ethyl-5-benzoyl-6-(benzylamino)-2-hydroxy-4oxohexa-2,5-dienoate (3o). Yield 43\% (0.181 g), yellow powder, mp 137-139 ${ }^{\circ} \mathrm{C}$. IR (ATR): 3241, 3032, 2987, 1729, 1593, 1258, $1021,907 \mathrm{~cm}^{-1}$. This compound exists in $\mathrm{CDCl}_{3}$ as a mixture of tautomers $E$-3o : $Z$-3o : keto-3o $=68: 19: 13 .{ }^{1} \mathrm{H}$ NMR $\left(\mathrm{CDCl}_{3}\right.$, ppm) $\delta: E-30(68 \%)-1.31(\mathrm{t}, J=7.1 \mathrm{~Hz}, 3 \mathrm{H}, \mathrm{Me}), 4.29$ (q, $J=$ $\left.7.1 \mathrm{~Hz}, 2 \mathrm{H}, \mathrm{OCH}_{2}\right), 4.54\left(\mathrm{~d}, J=6.0 \mathrm{~Hz}, 2 \mathrm{H}, \mathrm{CH}_{2} \mathrm{Ph}\right), 7.01(\mathrm{~s}, 1 \mathrm{H}$, $=\mathrm{CH}), 7.27\left(\mathrm{dd}, J=6.9 \mathrm{~Hz}, J=1.3 \mathrm{~Hz}, 2 \mathrm{H}, \mathrm{H}-2, \mathrm{H}-6 \mathrm{PhCH}_{2}\right.$ ), 7.31-7.52 (5H, m, $\left.P h \mathrm{CH}_{2}, \mathrm{Ph}\right), 7.53$ (t, $J=7.5 \mathrm{~Hz}, 1 \mathrm{H}, \mathrm{H}-4 \mathrm{Ph}$ ), 7.63 (dd, $J=7.8 \mathrm{~Hz}, J=1.4 \mathrm{~Hz}, 1 \mathrm{H}, \mathrm{H}-2, \mathrm{H}-6 \mathrm{Ph}), 7.69$ (d, $J=$ $13.7 \mathrm{~Hz}, 1 \mathrm{H},=\mathrm{CHN}), 10.95(\mathrm{dt}, J=13.7 \mathrm{~Hz}, J=6.0 \mathrm{~Hz}, 1 \mathrm{H}, \mathrm{NH})$, $14.43(\mathrm{~s}, 1 \mathrm{H}, \mathrm{OH})$. Anal. calcd for $\mathrm{C}_{22} \mathrm{H}_{21} \mathrm{NO}_{5}$ : C, 69.64; $\mathrm{H}$, 5.58; N, 3.69. Found: C, 69.48; H, 5.61; N, 3.97.

\section{General method for the preparation of polycarbonyl compounds 4 and 6}

Method A. Pyrone $1(0.26 \mathrm{mmol})$ was added to EtOH $(2 \mathrm{~mL})$ containing compound $3(0.26 \mathrm{mmol})$ under cooling on an ice bath. The resulted mixture was stirred at $0{ }^{\circ} \mathrm{C}$ for $1 \mathrm{~h}$ and then at $20^{\circ} \mathrm{C}$ for $24 \mathrm{~h}$. The precipitate was filtered and washed with $\mathrm{EtOH}$.

Method B. Pyrone $1(0.72 \mathrm{mmol})$ was added to EtOH $(4 \mathrm{~mL})$ containing diamine $2(0.36 \mathrm{mmol})$ under cooling on an ice bath. The resulted mixture was stirred at $0{ }^{\circ} \mathrm{C}$ for $1 \mathrm{~h}$ and then 
at $20{ }^{\circ} \mathrm{C}$ for $24 \mathrm{~h}$. The precipitate was filtered and washed with EtOH.

$\left(2 Z, 2^{\prime} Z, 5 E, 5^{\prime} E\right)$-Diethyl-6,6'-(1,2-phenylenebis(azanediyl)) bis(5-benzoyl-2-hydroxy-4-oxohexa-2,5-dienoate) (4a). Yield 64\% (0.109 g, Method A), 57\% (0.134 g, Method B), yellow powder, $\mathrm{mp}$ 148-150 ${ }^{\circ} \mathrm{C}$. IR (ATR): 2987, 1728, 1716, 1609, 1571, $1247 \mathrm{~cm}^{-1} ;{ }^{1} \mathrm{H} \mathrm{NMR}\left(\mathrm{CDCl}_{3}, \mathrm{ppm}\right) \delta:\left(5 E, 5^{\prime} E\right)-\mathbf{4 a}(50 \%)-1.34(\mathrm{t}, J$ $=7.1 \mathrm{~Hz}, 3 \mathrm{H}, \mathrm{Me}), 4.31\left(\mathrm{q}, J=7.1 \mathrm{~Hz}, 2 \mathrm{H}, \mathrm{OCH}_{2}\right), 6.94(\mathrm{~s}, 1 \mathrm{H},=$ $\mathrm{CH}$ ), 7.13-7.22 (m, 1H, Ar), 7.24-7.33 (m, 1H, Ar), 7.50 (2H, dd, $=7.9 \mathrm{~Hz}, J=7.2 \mathrm{~Hz}, \mathrm{H}-3, \mathrm{H}-5 \mathrm{Ph}), 7.55-7.66(\mathrm{~m}, 1 \mathrm{H}, \mathrm{H}-4 \mathrm{Ph})$, 7.75-7.81 (m, 2H, H-2, H-6 Ph), 7.98 (d, $J=12.5 \mathrm{~Hz}, 1 \mathrm{H},=\mathrm{CHN}$ ), $12.58(\mathrm{~d}, J=12.5 \mathrm{~Hz}, 1 \mathrm{H}, \mathrm{NH}), 14.18(\mathrm{~s}, 1 \mathrm{H}, \mathrm{OH}) ;{ }^{13} \mathrm{C} \mathrm{NMR}$ (DMSO- $\left.d_{6}, \mathrm{ppm}\right) \delta 14.0,62.1,103.7,113.2$, 120.3, 127.2, 129.1, 129.4, 131.5, 132.4, 139.4, 154.6, 160.8, 162.3, 193.9, 194.4. Anal. calcd for $\mathrm{C}_{36} \mathrm{H}_{32} \mathrm{~N}_{2} \mathrm{O}_{10}: \mathrm{C}, 66.25 ; \mathrm{H}, 4.94 ; \mathrm{N}, 4.29$. Found: $\mathrm{C}$, 66.38; H, 4.86; N, 4.29.

(2Z,2' Z,5E,5'E)-Diethyl-6,6'-(1,2-phenylenebis(azanediyl)) bis(2-hydroxy-5-(4-methoxybenzoyl)-4-oxohexa-2,5-dienoate) (4b). Yield 73\% (0.135 g, Method A), 38\% (0.098 g, Method B), yellow powder, $\mathrm{mp} 139-141^{\circ} \mathrm{C}$. IR (ATR): 3134, 2988, 2839, 1735, 1725, 1594, 1564, 1248, 1032, $839 \mathrm{~cm}^{-1} ;{ }^{1} \mathrm{H} \mathrm{NMR}\left(\mathrm{CDCl}_{3}, \mathrm{ppm}\right)$ $\delta$ : $\left(5 E, 5^{\prime} E\right)-4 \mathbf{b}(69 \%)-1.31(\mathrm{t}, J=7.1 \mathrm{~Hz}, 3 \mathrm{H}, \mathrm{Me}), 3.88(\mathrm{~s}, 3 \mathrm{H}$, $\mathrm{OMe}), 4.29$ (q, $\left.J=7.1 \mathrm{~Hz}, 2 \mathrm{H}, \mathrm{OCH}_{2}\right), 6.82(\mathrm{~s}, 1 \mathrm{H},=\mathrm{CH}), 6.96(\mathrm{~d}$, $\mathrm{AA}^{\prime} \mathrm{BB}^{\prime}, J=8.8 \mathrm{~Hz}, 2 \mathrm{H}, \mathrm{H}-3, \mathrm{H}-5 \mathrm{Ar}^{\prime}$ ), 7.14-7.30 (m, 2H, Ar), 7.77 (d, $\left.J=8.8 \mathrm{~Hz}, \mathrm{AA}^{\prime} \mathrm{BB}^{\prime}, 2 \mathrm{H}, \mathrm{H}-2, \mathrm{H}-6 \mathrm{Ar}^{\prime}\right), 7.92$ (d, $J=12.5 \mathrm{~Hz}, 1 \mathrm{H}$, $=\mathrm{CHN}), 12.49$ (d, $J=12.5 \mathrm{~Hz}, 1 \mathrm{H}, \mathrm{NH}), 14.18(\mathrm{~s}, 1 \mathrm{H}, \mathrm{OH}) ;{ }^{13} \mathrm{C}$ NMR (DMSO- $\left.d_{6}, \mathrm{ppm}\right) \delta 14.0,55.5,62.1,103.7,113.9,120.2$, $127.5,131.5,131.9$, 132.2, 153.6, 156.8, 160.6, 162.4, 163.3, 192.7, 194.2. Anal. calcd for $\mathrm{C}_{30} \mathrm{H}_{36} \mathrm{~N}_{2} \mathrm{O}_{12}$ : C, 64.04; H, 5.09; N, 3.93. Found: C, 63.78; H, 4.92; N, 4.05.

$\left(2 Z, 2^{\prime} Z, 5 E, 5^{\prime} E\right)$-Diethyl-6,6 $6^{\prime}$-(1,2-phenylenebis(azanediyl)) bis(5-(4-chlorobenzoyl)-2-hydroxy-4-oxohexa-2,5-dienoate) (4c). Yield 65\% (0.122 g, Method A), 75\% (0.195 g, Method B), yellow powder, mp 140-141 ${ }^{\circ} \mathrm{C}$. IR (ATR): 3147, 3063, 2987, 2924, 1737, 1724, 1613, $1253 \mathrm{~cm}^{-1} ;{ }^{1} \mathrm{H}$ NMR $\left(\mathrm{CDCl}_{3}, \mathrm{ppm}\right) \delta:\left(5 E, 5^{\prime} E\right)-4 \mathrm{c}$ $(39 \%)-1.32$ (t, $J=7.1 \mathrm{~Hz}, 3 \mathrm{H}, \mathrm{Me}), 4.29$ (q, $J=7.1 \mathrm{~Hz}, 2 \mathrm{H}$, $\left.\mathrm{OCH}_{2}\right), 6.85(\mathrm{~s}, 1 \mathrm{H},=\mathrm{CH}), 7.15-7.40(\mathrm{~m}, 2 \mathrm{H}, \mathrm{Ar}), 7.39-7.50(\mathrm{~m}$, $\left.2 \mathrm{H}, \mathrm{Ar}^{\prime}\right), 7.70$ (d, $\left.J=8.5 \mathrm{~Hz}, \mathrm{AA}^{\prime} \mathrm{BB}^{\prime}, 2 \mathrm{H}, \mathrm{H}-3, \mathrm{H}-5 \mathrm{Ar}^{\prime}\right), 7.93$ (d, $J=$ $12.6 \mathrm{~Hz}, 1 \mathrm{H},=\mathrm{CHN}), 12.55$ (d, $J=12.6 \mathrm{~Hz}, 1 \mathrm{H}, \mathrm{NH}), 14.09$ (s, $1 \mathrm{H}, \mathrm{OH}) .{ }^{13} \mathrm{C} \mathrm{NMR}\left(\mathrm{CDCl}_{3}, \mathrm{ppm}\right) \delta 14.0,62.2,103.6,113.0,120.3$, $127.9,129.0,129.8,130.8,137.6,138.8,154.5,160.9,162.2$, 192.5, 194.1. Anal. calcd for $\mathrm{C}_{36} \mathrm{H}_{30} \mathrm{Cl}_{2} \mathrm{~N}_{2} \mathrm{O}_{10}: \mathrm{C}, 59.93 ; \mathrm{H}$, 4.19; N, 3.88. Found: C, 59.64; H, 3.82; N, 4.15.

(2Z,2' Z,5E,5'E)-Diethyl-6,6' -(1,2-phenylenebis(azanediyl)) bis(2-hydroxy-4-oxo-5-(thiophene-2-carbonyl)hexa-2,5-dienoate) (4d). Yield 75\% (0.130 g, Method A), 31\% (0.074 g, Method B), yellow powder, $\mathrm{mp} 134-136^{\circ} \mathrm{C}$. IR (ATR): 3097, 2983, 1718, 1591, 1569, 1243, $842 \mathrm{~cm}^{-1} ;{ }^{1} \mathrm{H}$ NMR $\left(\mathrm{CDCl}_{3}, \mathrm{ppm}\right) \delta:\left(5 E, 5^{\prime} E\right)-4 \mathrm{~d}$ $(71 \%)-1.34(\mathrm{t}, J=7.1 \mathrm{~Hz}, 3 \mathrm{H}, \mathrm{Me}), 4.31(\mathrm{q}, J=7.1 \mathrm{~Hz}, 2 \mathrm{H}$, $\left.\mathrm{OCH}_{2}\right), 6.95(\mathrm{~s}, 1 \mathrm{H},=\mathrm{CH}), 7.15(\mathrm{dd}, J=4.8 \mathrm{~Hz}, J=3.8 \mathrm{~Hz}, 1 \mathrm{H}, \mathrm{H}-$ $4 \mathrm{Th}$ ), 7.23-7.33 (m, 2H, Ar), 7.59 (dd, $J=3.8 \mathrm{~Hz}, J=0.8 \mathrm{~Hz}, 1 \mathrm{H}$, H-3 Th), 7.70 (dd, $J=4.8 \mathrm{~Hz}, J=0.8 \mathrm{~Hz}, 1 \mathrm{H}, \mathrm{H}-5 \mathrm{Th}$ ), 8.09 (d, $J=$ $12.4 \mathrm{~Hz}, 1 \mathrm{H},=\mathrm{CHN}), 12.48$ (d, $J=12.4 \mathrm{~Hz}, 1 \mathrm{H}, \mathrm{NH}), 14.12$ (s, $1 \mathrm{H}, \mathrm{OH}) ;{ }^{13} \mathrm{C} \mathrm{NMR}\left(\mathrm{CDCl}_{3}, \mathrm{ppm}\right) \delta 14.1,62.2,103.2,113.5,120.4$, $127.6,128.1$, 131.5, 133.4, 134.0, 144.7, 153.0, 160.8, 162.3, 185.0, 193.7. Anal. calcd for $\mathrm{C}_{32} \mathrm{H}_{28} \mathrm{~N}_{2} \mathrm{O}_{10} \mathrm{~S}_{2}: \mathrm{C}, 57.82 ; \mathrm{H}, 4.25 ; \mathrm{N}$, 4.21. Found: C, 57.81; H, 4.20; N, 4.12.
(2Z,2 $\left.Z, 5 E, 5^{\prime} E\right)$-Diethyl-6,6'-(4,5-dimethyl-1,2-phenylene) bis(azanediyl)bis(2-hydroxy-4-oxo-5-(thiophene-2-carbonyl) hexa-2,5-dienoate) (4e). Yield 79\% (0.142 g, Method A), 63\% (0.157 g, Method B), red powder, mp $174-176{ }^{\circ} \mathrm{C}$. IR (ATR): 3148, 2984, 1742, 1726, 1610, 1566, 1411, 1238, $842 \mathrm{~cm}^{-1} ;{ }^{1} \mathrm{H}$ NMR $\left(\mathrm{CDCl}_{3}, \mathrm{ppm}\right) \delta:\left(5 E, 5^{\prime} E\right)-4 \mathrm{e}(74 \%)-1.33(\mathrm{t}, J=7.1 \mathrm{~Hz}, 3 \mathrm{H}$, $\mathrm{OCH}_{2} \mathrm{Me}$ ), 2.26 (s, 3H, Me), 4.31 (q, $\left.J=7.1 \mathrm{~Hz}, 2 \mathrm{H}, \mathrm{OCH}_{2}\right), 6.93$ $(\mathrm{s}, 1 \mathrm{H},=\mathrm{CH}), 7.00(\mathrm{br}, 1 \mathrm{H}, \mathrm{Ar}), 7.14(\mathrm{dd}, J=5.0 \mathrm{~Hz}, J=3.8 \mathrm{~Hz}$, 1H, H-4 Th), 7.57 (dd, $J=3.8 \mathrm{~Hz}, J=0.9 \mathrm{~Hz}, 1 \mathrm{H}, \mathrm{H}-3 \mathrm{Th}$ ), 7.69 $(\mathrm{dd}, J=5.0 \mathrm{~Hz}, J=0.9 \mathrm{~Hz}, 1 \mathrm{H}, \mathrm{H}-5 \mathrm{Th}), 8.05$ (d, $J=12.6 \mathrm{~Hz}, 1 \mathrm{H}$, $=\mathrm{CHN}), 12.41(\mathrm{~d}, J=12.6 \mathrm{~Hz}, 1 \mathrm{H}, \mathrm{NH}), 14.14(\mathrm{~s}, 1 \mathrm{H}, \mathrm{OH}) ;{ }^{13} \mathrm{C}$ $\mathrm{NMR}\left(\mathrm{CDCl}_{3}, \mathrm{ppm}\right) \delta 14.0,19.5,62.1,103.3,113.0,121.5,128.0$, 129.1, 133.4, 133.9, 136.9, 144.9, 153.4, 160.5, 162.4, 185.0, 193.5. Anal. calcd for $\mathrm{C}_{34} \mathrm{H}_{32} \mathrm{~N}_{2} \mathrm{O}_{10} \mathrm{~S}_{2}$ : C, 58.95; H, 4.66; N, 4.04 . Found: C, 58.90; H, 4.77; N, 4.05 .

\section{Binuclear copper(II) complex 5}

$\mathrm{Cu}(\mathrm{OAc})_{2}(0.028 \mathrm{~g}, 0.15 \mathrm{mmol})$ was added to solution of polycarbonyl compounds $4 \mathrm{c}(0.050 \mathrm{~g}, 0.069 \mathrm{mmol})$ in DMF (1 mL). The reaction mixture was stirred for $10 \mathrm{~min}$ at room temperature. The precipitate that formed was filtered and washed with EtOH. Yield $87 \%(0.051 \mathrm{~g})$, red-brown powder, $\mathrm{mp}>330{ }^{\circ} \mathrm{C} .{ }^{1} \mathrm{H}$ NMR (DMSO- $d_{6}, \mathrm{ppm}$ ) (selected signals) $\delta 1.10$ (br, $3 \mathrm{H}, \mathrm{Me}$ ), 3.76 (br, 2H, $\mathrm{CH}_{2}$ ), 5.74 (br, 1H, =CH), 7.24-7.80 (m, 6H, Ar). IR (ATR): 2982, 1733, 1723, 1713, 1631, 1569, 1338, 1630, 1393, 1340, 1247, 995, 911, 816, $749 \mathrm{~cm}^{-1}$. Anal. calcd for $\mathrm{C}_{36} \mathrm{H}_{26} \mathrm{Cl}_{2}$ $\mathrm{Cu}_{2} \mathrm{~N}_{2} \mathrm{O}_{10}: \mathrm{C}, 51.19 ; \mathrm{H}, 3.10 ; \mathrm{N}, 3.32$. Found: $\mathrm{C}, 51.06 ; \mathrm{H}, 3.11 ; \mathrm{N}$, 3.62. HRMS (ESI) calcd for $\mathrm{C}_{36} \mathrm{H}_{27} \mathrm{~N}_{2} \mathrm{O}_{10} \mathrm{Cl}_{2} \mathrm{Cu}_{2}[\mathrm{M}+\mathrm{H}]^{+}$ 842.9635 , found 842.9622 .

(2Z,2' $\left.Z, 5 E, 5^{\prime} E\right)$-Diethyl-6,6'-(1,4-phenylenebis(azanediyl)) bis(5-benzoyl-2-hydroxy-4-oxohexa-2,5-dienoate) (6). Yield 99\% (0.233 g, Method B), yellow powder, mp 178-180 ${ }^{\circ} \mathrm{C}$. IR (ATR): $3138,1729,1572,1454,1246,1102,834 \mathrm{~cm}^{-1}$; this compound exists in $\mathrm{CDCl}_{3}$ as a mixture of tautomers $\left(5 E, 5^{\prime} E\right)-6:\left(5 E, 5^{\prime} Z\right)$ 6 : keto- $\left(5 E, 5^{\prime} E\right)-6=84: 8: 8 .{ }^{1} \mathrm{H} \mathrm{NMR}\left(\mathrm{CDCl}_{3}, \mathrm{ppm}\right) \delta:\left(5 E, 5^{\prime} E\right)-$ $6(84 \%)-1.33(3 \mathrm{H}, \mathrm{t}, J=7.1 \mathrm{~Hz}, \mathrm{Me}), 4.31(2 \mathrm{H}, \mathrm{q}, J=7.1 \mathrm{~Hz}$, $\left.\mathrm{OCH}_{2}\right), 6.94(1 \mathrm{H}, \mathrm{s},=\mathrm{CH}), 7.15(2 \mathrm{H}, \mathrm{s}, \mathrm{Ar}), 7.50(\mathrm{dd}, J=7.8 \mathrm{~Hz}, J$ $=7.3 \mathrm{~Hz}, 2 \mathrm{H}, \mathrm{H}-3, \mathrm{H}-5 \mathrm{Ph}), 7.57-7.60(\mathrm{~m}, 1 \mathrm{H}, \mathrm{H}-4 \mathrm{Ph}), 7.75$ (d, $2 \mathrm{H}, J=7.3 \mathrm{~Hz}, \mathrm{H}-2, \mathrm{H}-6 \mathrm{Ph}), 8.02(\mathrm{~d}, J=13.2 \mathrm{~Hz}, 1 \mathrm{H},=\mathrm{CHN}$ ), $12.46(\mathrm{~d}, J=13.2 \mathrm{~Hz}, 1 \mathrm{H}, \mathrm{NH}), 14.27$ (s, 1H, OH). Anal. calcd for $\mathrm{C}_{36} \mathrm{H}_{32} \mathrm{~N}_{2} \mathrm{O}_{10}$ : C, 66.25; H, 4.94; N, 4.29. Found: C, 66.14; $\mathrm{H}$, $4.81 ; \mathrm{N}, 4.45$.

General approach for the preparation of $6 a$-hydroxy-9-acyl6a,7-dihydro-5 $H$-pyrido[1,2-a]quinoxaline-6,8-diones 7

Enaminone $\mathbf{3 e}, \mathbf{f}, \mathbf{h}, \mathbf{j}(0.259 \mathrm{mmol})$ was refluxed in EtOH $(2 \mathrm{~mL})$ for $20 \mathrm{~min}$. The precipitate that formed was filtered and washed with EtOH.

6a-Hydroxy-9-(4-methoxybenzoyl)-6a,7-dihydro-5H-pyrido [1,2-a]quinoxaline-6,8-dione (7a). Yield 72\% (0.0679 g), light yellow powder, $\mathrm{mp}>300{ }^{\circ} \mathrm{C}$. IR (ATR): 3067, 2915, 1692, 1677, 1598, 1541, 1248, 1174, $758 \mathrm{~cm}^{-1}$; ${ }^{1} \mathrm{H}$ NMR (DMSO- $\left.d_{6}, \mathrm{ppm}\right)$ $\delta 2.95(\mathrm{~d}, J=16.6 \mathrm{~Hz}, 1 \mathrm{H}, \mathrm{CHH}), 3.40(\mathrm{~d}, J=16.7 \mathrm{~Hz}, 1 \mathrm{H}, \mathrm{CH} H)$, 3.84 (s, 3H, MeO), 6.99 (d, $\mathrm{AA}^{\prime} \mathrm{BB}^{\prime}, J=8.9 \mathrm{~Hz}, 2 \mathrm{H}, \mathrm{H}-3, \mathrm{H}-5 \mathrm{Ar}$ ), $7.00-7.13(\mathrm{~m}, 2 \mathrm{H}), 7.18(\mathrm{td}, J=7.9 \mathrm{~Hz}, 1 \mathrm{H}), 7.54(\mathrm{~d}, J=7.9 \mathrm{~Hz}$, 1H), 7.71 (d, $\left.\mathrm{AA}^{\prime} \mathrm{BB}^{\prime}, J=8.6 \mathrm{~Hz}, 2 \mathrm{H}, \mathrm{H}-2, \mathrm{H}-6 \mathrm{Ar}\right), 7.76$ (s, 1H, 
$\mathrm{OH}), 8.41(\mathrm{~s}, 1 \mathrm{H}, \mathrm{H}-10), 11.08(\mathrm{~s}, 1 \mathrm{H}, \mathrm{NH})$. Anal. calcd for $\mathrm{C}_{20} \mathrm{H}_{16} \mathrm{~N}_{2} \mathrm{O}_{5}$ : C, 66.01; H, 7.36; N, 4.41. Found: C, 65.93; H, $7.69 ; \mathrm{N}, 4.43$.

9-(4-Chlorobenzoyl)-6a-hydroxy-6a,7-dihydro-5H-pyrido[1,2a]quinoxaline-6,8-dione (7b). Yield 89\% (0.0850 g), yellow powder, $\mathrm{mp}>320^{\circ} \mathrm{C}$. IR (ATR): 3132, 3061, 3988, 2906, 2870, 2763, 1696, 1679, 1621, 1543, 1250, $749 \mathrm{~cm}^{-1}$; ${ }^{1} \mathrm{H}$ NMR (DMSO$\left.d_{6}, \mathrm{ppm}\right) \delta 2.97(\mathrm{~d}, J=16.7 \mathrm{~Hz}, 1 \mathrm{H}, \mathrm{CHH}), 3.30(\mathrm{~d}, J=16.7 \mathrm{~Hz}$, 1H, CHH), 7.04-7.12 (m, 2H, Ar), 7.14-7.19 (m, 1H), 7.45 (d, $\left.\mathrm{AA}^{\prime} \mathrm{BB}^{\prime}, J=8.5 \mathrm{~Hz}, 2 \mathrm{H}, \mathrm{H}-2, \mathrm{H}-6 \mathrm{Ar}\right), 7.50$ (d, $\left.J=7.9 \mathrm{~Hz}, 1 \mathrm{H}\right), 7.65$ $\left(2 \mathrm{H}, \mathrm{d}, \mathrm{AA}^{\prime} \mathrm{BB}^{\prime}, J=8.6 \mathrm{~Hz}, \mathrm{H}-3, \mathrm{H}-5 \mathrm{Ar}\right), 7.75(\mathrm{~s}, 1 \mathrm{H}, \mathrm{OH}), 8.46$ (s, $1 \mathrm{H}, \mathrm{H}-10), 11.02$ (s, $1 \mathrm{H}, \mathrm{NH}) ;{ }^{13} \mathrm{C}$ NMR (DMSO- $\left.d_{6}, \mathrm{ppm}\right) \delta 45.0$, 82.5, 114.0, 116.3, 117.3, 123.5, 125.2, 125.7, 127.9, 128.3, 130.9, $136.7,137.8,149.4,163.3,187.2$, 190.1. Anal. calcd for $\mathrm{C}_{19} \mathrm{H}_{13} \mathrm{ClN}_{2} \mathrm{O}_{4}: \mathrm{C}, 61.88 ; \mathrm{H}, 3.55 ; \mathrm{N}, 7.60$. Found: C, 61.65; H, $3.25 ; \mathrm{N}, 7.77$.

6a-Hydroxy-9-(thiophene-2-carbonyl)-6a,7-dihydro-5H-pyrido[1,2-a]quinoxaline-6,8-dione (7c). Yield 86\% (0.0758 g), beige powder, mp $>320{ }^{\circ} \mathrm{C} .{ }^{1} \mathrm{H}$ NMR $\left(\mathrm{DMSO}-d_{6}, \mathrm{ppm}\right) \delta 3.01(\mathrm{~d}, J=$ $16.6 \mathrm{~Hz}, 1 \mathrm{H}, \mathrm{CHH}), 3.38$ (d, $J=16.6 \mathrm{~Hz}, 1 \mathrm{H}, \mathrm{CH} H), 7.05-7.14(\mathrm{~m}$, 2H, Ar), 7.15-7.23 (m, 2H, Th, Ar), 7.54 (d, $J=8.0 \mathrm{~Hz}, 1 \mathrm{H}), 7.50$ $(\mathrm{d}, J=7.9 \mathrm{~Hz}, 1 \mathrm{H}), 7.71-7.80(\mathrm{~m}, 2 \mathrm{H}, \mathrm{OH}, \mathrm{Th}), 7.95$ (d, 1H, $J=$ $4.8 \mathrm{~Hz}, \mathrm{H}-5 \mathrm{Th}$ ), 8.49 (s, 1H, H-10), 11.07 (s, 1H, NH); ${ }^{13} \mathrm{C}$ NMR $\left(\mathrm{DMSO}-d_{6}, \mathrm{ppm}\right) \delta 45.1,82.3,114.5,116.3,117.2,123.5,125.35$, 125.44, 128.2 (2C), 134.2, 134.6, 144.5, 148.1, 163.3, 182.5, 187.0. Anal. calcd for $\mathrm{C}_{17} \mathrm{H}_{10} \mathrm{~N}_{2} \mathrm{O}_{3} \mathrm{~S}$ : C, 59.99; H, 3.55; N, 8.23. Found: C, 59.73; H, 3.58; N, 8.24.

6a-Hydroxy-2,3-dimethyl-9-(thiophene-2-carbonyl)-6a,7dihydro-5H-pyrido[1,2-a]quinoxaline-6,8-dione (7d). Yield 85\% (0.0811 g), orange powder, $\mathrm{mp}>300{ }^{\circ} \mathrm{C}$. IR (ATR): 3179, 3082, 2976, 1722, 1674, 1591, 1539, 1514, 1312, 1044, $841 \mathrm{~cm}^{-1} ;{ }^{1} \mathrm{H}$ NMR (DMSO- $\left.d_{6}, \mathrm{ppm}\right) \delta 2.18$ (s, 3H, Me), 2.20 (s, 3H, Me), 2.96 $(\mathrm{d}, J=16.7 \mathrm{~Hz}, 1 \mathrm{H}, \mathrm{CHH}), 3.36(\mathrm{~d}, J=16.7 \mathrm{~Hz}, 1 \mathrm{H}, \mathrm{CH}), 6.81(\mathrm{~s}$, $1 \mathrm{H}), 7.20$ (dd, $J=4.9 \mathrm{~Hz}, J=3.9 \mathrm{~Hz}, 1 \mathrm{H}, \mathrm{H}-4 \mathrm{Th}), 7.37$ (s, 1H), 7.70 (s, 1H, OH), 7.75 (dd, $J=3.9 \mathrm{~Hz}, J=1.0 \mathrm{~Hz}, 1 \mathrm{H}, \mathrm{H}-3 \mathrm{Th}$ ), 7.95 (dd, $J=4.9 \mathrm{~Hz}, J=1.0 \mathrm{~Hz}, 1 \mathrm{H}, \mathrm{H}-5 \mathrm{Th}), 8.47$ (s, 1H, H-10), 10.93 (s, $1 \mathrm{H}, \mathrm{NH}) ;{ }^{13} \mathrm{C}$ NMR (DMSO- $\left.d_{6}, \mathrm{ppm}\right) \delta 18.8$ (2Me), 45.1, 82.4, 114.2, 117.0, 117.9, 123.0, 125.8, 128.1, 131.6, 133.6, 134.1, $134.7,144.6,148.1,163.3,182.5,186.8$. Anal. calcd for $\mathrm{C}_{19} \mathrm{H}_{16} \mathrm{~N}_{2} \mathrm{O}_{4} \mathrm{~S}: \mathrm{C}, 61.94 ; \mathrm{H}, 4.38 ; \mathrm{N}, 7.60$. Found: C, 61.95; H, $4.35 ; \mathrm{N}, 7.74$.

General approach for the preparation of 9-acyl-5H-pyrido[1,2a]quinoxaline-6,8-diones 8

Compound $7(0.147 \mathrm{mmol})$ was refluxed in $\mathrm{AcOH}(2 \mathrm{~mL})$ for $20 \mathrm{~min}$. After cooling, the reaction mixture was diluted with $\mathrm{H}_{2} \mathrm{O}(3 \mathrm{~mL})$. The precipitate that formed was filtered and washed with cold EtOH. The product was dried at $100{ }^{\circ} \mathrm{C}$.

9-(4-Methoxybenzoyl)-5H-pyrido[1,2-a]quinoxaline-6,8-dione (8a). Yield 89\% (0.0453 g), grey powder, $\mathrm{mp}>300{ }^{\circ} \mathrm{C}$. IR (ATR): 3677, 2973, 2901, 1687, 1683, 1605, 1591, 1575, 1242, 1017, $850 \mathrm{~cm}^{-1} ;{ }^{1} \mathrm{H}$ NMR (DMSO- $\left.d_{6}, \mathrm{ppm}\right) \delta 3.85(\mathrm{~s}, 3 \mathrm{H}, \mathrm{Me}), 7.05(\mathrm{~d}$, $\mathrm{AA}^{\prime} \mathrm{BB}^{\prime}, J=8.9 \mathrm{~Hz}, 2 \mathrm{H}, \mathrm{H}-3, \mathrm{H}-5 \mathrm{Ar}$ ), 7.12 (s, 1H, H-7), 7.24 (ddd, $J$ $=8.0 \mathrm{~Hz}, J=7.6 \mathrm{~Hz}, J=1.4 \mathrm{~Hz}, 1 \mathrm{H}, \mathrm{H}-2), 7.29(\mathrm{dd}, J=8.0 \mathrm{~Hz}, J=$ $1.4 \mathrm{~Hz}, 1 \mathrm{H}, \mathrm{H}-4), 7.38$ (td, $J=7.6 \mathrm{~Hz}, J=0.6 \mathrm{~Hz}, 1 \mathrm{H}, \mathrm{H}-3), 7.84$ $\left(\mathrm{d}, \mathrm{AA}^{\prime} \mathrm{BB}^{\prime}, J=8.6 \mathrm{~Hz}, 2 \mathrm{H}, \mathrm{H}-2, \mathrm{H}-6 \mathrm{Ar}\right), 8.20(\mathrm{dd}, J=8.6 \mathrm{~Hz}, J=$ $0.8 \mathrm{~Hz}, 1 \mathrm{H}, \mathrm{H}-1$ ), 9.12 (s, 1H, H-10), 12.06 (s, 1H, NH); ${ }^{13} \mathrm{C}$ NMR
$\left(\mathrm{DMSO}-d_{6}, \mathrm{ppm}\right) \delta 56.0,113.9,115.5,117.0,117.2,123.1,123.7$, $127.1,127.5,129.5,131.9,132.6,133.4,137.5,154.7,163.6$, 174.6, 191.8. Anal. calcd for $\mathrm{C}_{20} \mathrm{H}_{14} \mathrm{~N}_{2} \mathrm{O}_{5}: \mathrm{C}, 69.36 ; \mathrm{H}, 4.07 ; \mathrm{N}$, 8.09. Found: C, 69.51; H, 4.41; N, 8.35.

9-(4-Chlorobenzoyl)-5H-pyrido[1,2-a]quinoxaline-6,8-dione (8b). Yield 89\% (0.0459 g), beige powder, $\mathrm{mp}>300{ }^{\circ} \mathrm{C}$. IR (ATR): 3063, 3036, 2987, 2905, 2841, 1710, 1241, $838 \mathrm{~cm}^{-1}$; ${ }^{1} \mathrm{H}$ NMR $\left(\right.$ DMSO- $\left.d_{6}, \mathrm{ppm}\right) \delta 7.15(\mathrm{~s}, 1 \mathrm{H}, \mathrm{H}-7), 7.23(\mathrm{td}, J=7.8 \mathrm{~Hz}, J=$ $1.6 \mathrm{~Hz}, 1 \mathrm{H}, \mathrm{H}-2), 7.30$ (dd, $J=7.8 \mathrm{~Hz}, J=1.6 \mathrm{~Hz}, 1 \mathrm{H}, \mathrm{H}-4), 7.36$ $(\mathrm{td}, J=7.5 \mathrm{~Hz}, J=0.6 \mathrm{~Hz}, 1 \mathrm{H}, \mathrm{H}-3), 7.55\left(\mathrm{~d}, \mathrm{AA}^{\prime} \mathrm{BB}^{\prime}, J=8.6 \mathrm{~Hz}\right.$, $2 \mathrm{H}, \mathrm{H}-3, \mathrm{H}-5 \mathrm{Ar}$ ), 7.84 (d, $\mathrm{AA}^{\prime} \mathrm{BB}^{\prime}, J=8.6 \mathrm{~Hz}, 2 \mathrm{H}, \mathrm{H}-2, \mathrm{H}-6 \mathrm{Ar}$ ), 8.16 (d, $J=8.5,1 \mathrm{H}, \mathrm{H}-1), 9.14$ (s, 1H, H-10), 12.04 (s, 1H, NH). Anal. calcd for $\mathrm{C}_{19} \mathrm{H}_{11} \mathrm{ClN}_{2} \mathrm{O}_{3}$ : C, 65.06; H, 3.16; N, 7.99. Found: C, 64.99; H, 3.38; N, 7.93.

9-(Thiophene-2-carbonyl)-5H-pyrido[1,2-a]quinoxaline-6,8dione (8c). Yield 92\% (0.0436 g), beige powder, $\mathrm{mp}>300{ }^{\circ} \mathrm{C}$. IR (ATR): 3236, 3099, 2922, 1705, 1701, 1561, 1380, $900 \mathrm{~cm}^{-1} ;{ }^{1} \mathrm{H}$ NMR (DMSO- $d_{6}$, ppm) $\delta 7.14(\mathrm{~s}, 1 \mathrm{H}, \mathrm{H}-7), 7.22-7.26(\mathrm{~m}, 2 \mathrm{H}, \mathrm{H}-4$ Th, H-2), 7.29 (1H, dd, $J=8.0 \mathrm{~Hz}, J=1.3 \mathrm{~Hz}, \mathrm{H}-4), 7.38$ (1H, td, $J$ $=7.5 \mathrm{~Hz}, J=0.5 \mathrm{~Hz}, \mathrm{H}-3), 7.74(\mathrm{dd}, J=3.8 \mathrm{~Hz}, J=1.0 \mathrm{~Hz}, 1 \mathrm{H}, \mathrm{H}-$ $3 \mathrm{Th}), 8.11$ (dd, $J=4.5 \mathrm{~Hz}, J=1.0 \mathrm{~Hz}, 1 \mathrm{H}, \mathrm{H}-5 \mathrm{Th}), 8.19$ (d, 1H, $=8.5 \mathrm{~Hz}, \mathrm{H}-1), 9.20(\mathrm{~s}, 1 \mathrm{H}, \mathrm{H}-10), 12.07$ (s, 1H, NH); ${ }^{13} \mathrm{C} \mathrm{NMR}$ $\left(\mathrm{DMSO}-d_{6}, \mathrm{ppm}\right) \delta 115.5,117.0,117.5,123.1,123.7,127.2,127.5$, 128.8, 131.7, 133.7, 136.1, 136.2, 137.5, 143.7, 154.6, 174.2, 185.0. Anal. calcd for $\mathrm{C}_{17} \mathrm{H}_{10} \mathrm{~N}_{2} \mathrm{O}_{3} \mathrm{~S}$ : C, 63.34; H, 3.13; N, 8.69. Found: C, 63.32; H, 3.38; N, 8.57.

2,3-Dimethyl-9-(thiophene-2-carbonyl)-5H-pyrido[1,2-a] quinoxaline-6,8-dione (8d). Yield 80\% (0.0412 g), light yellow powder, $\mathrm{mp}>300{ }^{\circ} \mathrm{C}$. IR (ATR): 3241, 3177, 3073, 2943, 1693, 1642, 1605, 1351, 1250, $848 \mathrm{~cm}^{-1}$; ${ }^{1} \mathrm{H}$ NMR (DMSO- $d_{6}, \mathrm{ppm}$ ) $\delta 2.23(\mathrm{~s}, 3 \mathrm{H}, \mathrm{Me}), 2.27$ (s, 3H, Me), $7.02(\mathrm{~s}, 1 \mathrm{H}), 7.16(\mathrm{~s}, 1 \mathrm{H}), 7.24$ $(\mathrm{dd}, J=4.5 \mathrm{~Hz}, J=3.2 \mathrm{~Hz}, 1 \mathrm{H}, \mathrm{H}-4 \mathrm{Th}), 7.76$ (d, $J=3.2 \mathrm{~Hz}, 1 \mathrm{H}$, H-3 Th), 7.97 (s, 1H, H-1), 8.07 (d, J=4.5 Hz, 1H, H-5 Th), 9.10 (s, $1 \mathrm{H}, \mathrm{H}-10), 11.80(\mathrm{~s}, 1 \mathrm{H}, \mathrm{NH})$. Anal. calcd for $\mathrm{C}_{19} \mathrm{H}_{14} \mathrm{~N}_{2} \mathrm{O}_{3} \mathrm{~S}: \mathrm{C}$, 65.13; H, 4.03; N, 7.99; H, 4.93. Found: C, 65.17; H, 3.94; N, 8.08.

Ethyl-5-benzoyl-4-oxo-1-phenyl-1,4-dihydropyridine-2-

carboxylate (9a). Enaminone $3 \mathbf{a}(0.100 \mathrm{~g}, 0.274 \mathrm{mmol})$ and $\mathrm{MeSO}_{3} \mathrm{H}(0.0526 \mathrm{~g}, 0.547 \mathrm{mmol})$ were stirred in $\mathrm{CH}_{2} \mathrm{Cl}_{2}(2 \mathrm{~mL})$ for $24 \mathrm{~h}$. The solvent was evaporated, and the residue was diluted with $\mathrm{H}_{2} \mathrm{O}(5 \mathrm{~mL})$. The precipitate that formed was filtered and was recrystallized from toluene-hexane. Yield $78 \%$ (0.074 g), white powder, mp 112-114 ${ }^{\circ} \mathrm{C}$. IR (ATR): 3055, 2970,

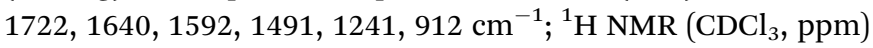
$\delta 1.09(\mathrm{t}, J=7.1 \mathrm{~Hz}, 3 \mathrm{H}, \mathrm{Me}), 4.13\left(\mathrm{q}, J=7.1 \mathrm{~Hz}, 2 \mathrm{H}, \mathrm{OCH}_{2}\right), 7.06$ (s, 1H, H-3), 7.30-7.36 (m, 2H, H-2, H-6 Ph), 7.45 (t, $J=7.6 \mathrm{~Hz}$, 2H, H-3, H-5 Ph), 7.48-7.54 (m, 3H, Ph, $\left.\mathrm{Ph}^{\prime}\right), 7.57$ (tt, $J=7.4 \mathrm{~Hz}, J$ $\left.=1.9 \mathrm{~Hz}, 1 \mathrm{H}, \mathrm{H}-4 \mathrm{Ph}^{\prime}\right), 7.89(\mathrm{dd}, J=8.5 \mathrm{~Hz}, J=1.4 \mathrm{~Hz}, 2 \mathrm{H}, \mathrm{H}-2$, H-6 $\mathrm{Ph}^{\prime}$ ), 7.91 (s, 1H, H-6). Anal. calcd for $\mathrm{C}_{21} \mathrm{H}_{17} \mathrm{NO}_{4}$ : C, 72.61; H, 4.93; N, 4.03. Found: C, 72.33; H, 4.81; N, 4.32.

Ethyl-5-(4-chlorobenzoyl)-1-(2-hydroxyphenyl)-4-oxo-1,4dihydropyridine-2-carboxylate (9b). Compound 3d (0.300 g, $0.721 \mathrm{mmol}$ ) was stirred in $\mathrm{EtOH}(9 \mathrm{~mL})$ containing concd $\mathrm{HCl}$ $(0.2 \mathrm{~mL})$ for $12 \mathrm{~h}$ at room temperature. The precipitate that formed was filtered. Yield $79 \%(0.227 \mathrm{~g})$, light white powder, $\mathrm{mp}$ 245-246 ${ }^{\circ}$ C. IR (ATR): 3060, 2983, 2902, 2740, 2576, 1728, 1665, $1632,1585,1552,1528 \mathrm{~cm}^{-1} ;{ }^{1} \mathrm{H}$ NMR (DMSO- $\left.d_{6}, \mathrm{ppm}\right) \delta 1.05(\mathrm{t}$, $J=7.1 \mathrm{~Hz}, 3 \mathrm{H}, \mathrm{Me}), 4.09$ (q, $\left.J=7.1 \mathrm{~Hz}, 2 \mathrm{H}, \mathrm{OCH}_{2}\right), 6.77(\mathrm{~s}, 1 \mathrm{H}$, 
$\mathrm{H}-3), 6.91(\mathrm{td}, J=7.7 \mathrm{~Hz}, J=1.2 \mathrm{~Hz}, 1 \mathrm{H}, \mathrm{H}-5 \mathrm{Ar}), 6.97$ (dd, $J=$ $8.2 \mathrm{~Hz}, J=1.1 \mathrm{~Hz}, 1 \mathrm{H}, \mathrm{H}-3 \mathrm{Ar}), 7.26(\mathrm{td}, J=8.0 \mathrm{~Hz}, J=1.1 \mathrm{~Hz}$, $1 \mathrm{H}, \mathrm{H}-4 \mathrm{Ar}$ ), 7.33 (dd, $J=7.8 \mathrm{~Hz}, J=1.4 \mathrm{~Hz}, 1 \mathrm{H}, \mathrm{H}-6 \mathrm{Ar}$ ), 7.49 (d, $\left.\mathrm{AA}^{\prime} \mathrm{BB}^{\prime}, J=8.4 \mathrm{~Hz}, 2 \mathrm{H}, \mathrm{H}-3, \mathrm{H}-5 \mathrm{Ar}^{\prime}\right), 7.77\left(\mathrm{~d}, \mathrm{AA}^{\prime} \mathrm{BB}^{\prime}, J=8.4 \mathrm{~Hz}\right.$, 2H, H-2, H-6 Ar'), 7.89 (s, 1H, H-6), 10.35 (s, 1H, OH). Anal. calcd for $\mathrm{C}_{21} \mathrm{H}_{16} \mathrm{ClNO}_{5}$ : C, 63.40; $\mathrm{H}, 4.05 ; \mathrm{N}, 3.52$. Found: $\mathrm{C}, 63.12 ; \mathrm{H}$, $4.04 ; \mathrm{N}, 3.73$.

(2E,4Z)-2-((2-Hydroxyphenylamino)methylene)-4-(2-oxo- $2 \mathrm{H}$ benzo $[b][1,4]$ oxazin-3(4H)-ylidene)-1-phenylbutane-1,3-dione

(10). Enaminone $3 \mathrm{c}(0.100 \mathrm{~g}, 0.262 \mathrm{mmol})$ and $o$-aminophenol $(0.030 \mathrm{~g}, 0.275 \mathrm{mmol})$ were refluxed in $\mathrm{C}_{6} \mathrm{H}_{6}(3 \mathrm{~mL})$ for $9 \mathrm{~h}$. The precipitate that formed was filtered. Yield $85 \%(0.095 \mathrm{mg})$, light white powder, $\mathrm{mp} 188-189^{\circ} \mathrm{C}$. IR (ATR): 3169, 3151, 3115, 3069, 2744, 2703, 1671, 1628, 1543, $1382 \mathrm{~cm}^{-1} ;{ }^{1} \mathrm{H}$ NMR $\left(\mathrm{DMSO}-d_{6}, \mathrm{ppm}\right) \delta 6.63(\mathrm{~s}, 1 \mathrm{H},=\mathrm{CH}), 6.68(\mathrm{t}, J=7.6 \mathrm{~Hz}, 1 \mathrm{H}$, Ar), 6.80-6.87 (m, 2H, Ar), 6.93 (t, $J=6.9 \mathrm{~Hz}, 1 \mathrm{H}, \mathrm{Ar}), 6.97$ (d, $=8.1 \mathrm{~Hz}, 1 \mathrm{H}, \mathrm{Ar}), 7.22(\mathrm{t}, J=7.7 \mathrm{~Hz}, 1 \mathrm{H}, \mathrm{Ar}), 7.42(\mathrm{~d}, J=7.8 \mathrm{~Hz}$, 1H, Ar), 7.44-7.51 (m, 3H, Ar, Ph), 7.59 (t, $J=7.3 \mathrm{~Hz}, 1 \mathrm{H}, \mathrm{H}-4$ $\mathrm{Ph}), 7.77$ (s, 1H, =CHN), $7.83(2 \mathrm{H}, \mathrm{d}, J=7.7 \mathrm{~Hz}, \mathrm{H}-2, \mathrm{H}-6 \mathrm{Ph})$, 9.56 (br, 1H, NH), 9.70 (s, 1H, OH), 10.25 (br, 1H, NH). Anal. calcd for $\mathrm{C}_{25} \mathrm{H}_{18} \mathrm{~N}_{2} \mathrm{O}_{5}: \mathrm{C}, 70.42 ; \mathrm{H}, 4.25 ; \mathrm{N}, 6.57$. Found: C, 70.09; H, 4.09; N, 6.57.

6a-Hydroxy-9-(thiophene-2-carbonyl)-6a,7-dihydro-5H-dipyrido $\left[1,2-a: 2^{\prime}, 3^{\prime}-e\right]$ pyrazine-6,8-dione (11). General approach for the preparation of enaminodiones 3 from ethyl 4-oxo-5-(thiophene-2-carbonyl)-4H-pyran-2-carboxylate (1e) and 2,3-diaminopyridine. Yield 91\% (0.345 g), dark grey powder, $\mathrm{mp}>$ $300{ }^{\circ} \mathrm{C}$. IR (ATR): 3153, 1703, 1657, 1608, 1557, 1406, 1328, 1038, $842 \mathrm{~cm}^{-1} ;{ }^{1} \mathrm{H}$ NMR (DMSO- $\left.d_{6}, \mathrm{ppm}\right) \delta 3.00(\mathrm{~d}, J=16.6 \mathrm{~Hz}, 1 \mathrm{H}$, $\mathrm{CHH}), 3.42$ (d, $J=16.6 \mathrm{~Hz}, 1 \mathrm{H}, \mathrm{CH} H), 7.15(\mathrm{dd}, J=8.1 \mathrm{~Hz}, J=$ $4.8 \mathrm{~Hz}, 1 \mathrm{H}, \mathrm{H}-2), 7.21$ (dd, $J=4.9 \mathrm{~Hz}, J=3.8 \mathrm{~Hz}, 1 \mathrm{H}, \mathrm{H}-4 \mathrm{Th}$ ), 7.74 (dd, $J=3.8 \mathrm{~Hz}, J=1.1 \mathrm{~Hz}, 1 \mathrm{H}, \mathrm{H}-3 \mathrm{Th}$ ), $7.80-7.98$ (s, $1 \mathrm{H}$, $\mathrm{OH}), 7.98(\mathrm{dd}, 1 \mathrm{H}, J=4.9, J=1.1, \mathrm{H}-5 \mathrm{Th}), 8.03(\mathrm{dd}, J=8.5 \mathrm{~Hz}, J$ $=0.8 \mathrm{~Hz}, 1 \mathrm{H}, \mathrm{H}-1), 8.12(1 \mathrm{H}, \mathrm{dd}, J=4.9, J=1.1, \mathrm{H}-3), 8.50$ (1H, s, H-10), $11.60(1 \mathrm{H}, \mathrm{s}, \mathrm{NH}) ;{ }^{13} \mathrm{C}$ NMR (DMSO- $\left.d_{6}, \mathrm{ppm}\right)$ $\delta 44.9,82.3,115.6,119.2,121.6,124.6,128.3,134.5,134.9,141.5$, 143.7, 144.4, 147.2, 164.1, 182.5, 187.0. Anal. calcd for $\mathrm{C}_{16} \mathrm{H}_{11} \mathrm{~N}_{3} \mathrm{O}_{4} \mathrm{~S}: \mathrm{C}, 56.30 ; \mathrm{H}, 3.25 ; \mathrm{N}, 12.31$. Found: C, 56.12; H, $3.31 ; \mathrm{N}, 12.08$.

9-(Thiophene-2-carbonyl)-5H-dipyrido[1,2-a:2' $\left.{ }^{\prime} 3^{\prime}-e\right]$ pyrazine6,8-dione (12). General approach for the preparation of compounds 8. Yield 81\% (0.0406 g), grey powder, $\mathrm{mp}>300{ }^{\circ} \mathrm{C}$. IR (ATR): 3075, 2744, 1704, 1623, 1547, 1256, $802 \mathrm{~cm}^{-1} ;{ }^{1} \mathrm{H}$ NMR (DMSO- $d_{6}$, ppm) $\delta 7.12(\mathrm{~s}, 1 \mathrm{H}, \mathrm{H}-7), 7.25$ (dd, $J=4.7 \mathrm{~Hz}, J=$ $3.7 \mathrm{~Hz}, 1 \mathrm{H}, \mathrm{H}-4 \mathrm{Th}$ ), 7.31 (dd, $J=8.4 \mathrm{~Hz}, J=4.7 \mathrm{~Hz}, 1 \mathrm{H}, \mathrm{H}-2$ ), $7.73(\mathrm{dd}, J=3.7 \mathrm{~Hz}, J=0.7 \mathrm{~Hz}, 1 \mathrm{H}, \mathrm{H}-3 \mathrm{Th}$ ), 8.12 (dd, $J=$ $4.7 \mathrm{~Hz}, J=0.7 \mathrm{~Hz}, 1 \mathrm{H}, \mathrm{H}-5 \mathrm{Th}$ ), 8.33 (dd, $J=4.7 \mathrm{~Hz}, J=0.7 \mathrm{~Hz}$, $1 \mathrm{H}, \mathrm{H}-3), 8.59$ (dd, $J=8.4 \mathrm{~Hz}, J=0.7 \mathrm{~Hz}, 1 \mathrm{H}, \mathrm{H}-1), 9.18(\mathrm{~s}, 1 \mathrm{H}, \mathrm{H}-$ 10), 12.52 (s, 1H, NH), $12.26(\mathrm{~s}, 1 \mathrm{H}, \mathrm{OH}) ;{ }^{13} \mathrm{C}$ NMR (DMSO- $d_{6}$, ppm) $\delta 117.4,119.3,120.0,123.6,128.9,131.8,133.8,136.31$, 136.34, 137.3, 140.6, 143.6, 146.0, 155.9, 174.4, 184.9. Anal. calcd for $\mathrm{C}_{16} \mathrm{H}_{9} \mathrm{~N}_{3} \mathrm{O}_{3} \mathrm{~S} \cdot \mathrm{H}_{2} \mathrm{O}: \mathrm{C}, 56.30 ; \mathrm{H}, 3.25 ; \mathrm{N}, 12.31$. Found: C, 56.31; H, 2.95; N, 12.12.

\section{Conflicts of interest}

There are no conflicts to declare.

\section{Acknowledgements}

This work was financially supported by the Russian Science Foundation (Grant 18-13-00186). Analytical studies were carried out using equipment of the Center for Joint Use "Spectroscopy and Analysis of Organic Compounds" at the Postovsky Institute of Organic Synthesis of the Russian Academy of Sciences (Ural Branch) and the Laboratory of Complex Investigations and Expert Evaluation of Organic Materials of the Center for Joint Use at the Ural Federal University. We also thank both reviewers for helpful suggestions.

\section{Notes and references}

1 (a) P. R. Christensen, A. M. Scheuermann, K. E. Loeffler and B. A. Helms, Nat. Chem., 2019, 11, 442; (b) E. A. Shokova, J. K. Kim and V. V. Kovalev, Russ. J. Org. Chem., 2015, 51, 755.

2 (a) N. V. Zolotareva and V. V. Semenov, Russ. Chem. Rev., 2013, 82, 964; (b) P. A. Vigato, V. Peruzzo and S. Tamburini, Coord. Chem. Rev., 2009, 253, 1099; (c) S. N. Podyachev, S. N. Sudakova, G. S. Gimazetdinova, N. A. Shamsutdinova, V. V. Syakaev, T. A. Barsukova, N. Iki, D. V. Lapaev and A. R. Mustafina, New J. Chem., 2017, 41, 1526; (d) A. J. Brock, J. K. Clegg, F. Li and L. F. Lindoy, Coord. Chem. Rev., 2018, 375, 106; (e) D. N. Bazhin, Y. S. Kudyakova, A. S. Bogomyakov, P. A. Slepukhin, G. A. Kim, Y. V. Burgart and V. I. Saloutin, Inorg. Chem. Front., 2019, 6, 40; (f) R. Pettinari, F. Marchetti, C. Di Nicola and C. Pettinari, Eur. J. Inorg. Chem., 2018, 31, 3521.

3 See reviews: (a) K. C. Gupta and A. K. Sutar, Coord. Chem. Rev., 2008, 252, 1420; (b) P. A. Vigato, V. Peruzzo and S. Tamburini, Coord. Chem. Rev., 2012, 256, 953; (c) C. J. Whiteoak, G. Salassa and A. W. Kleij, Chem. Soc. Rev., 2012, 41, 622; (d) C. Freire, M. Nunes, C. Pereira, D. M. Fernandes, A. F. Peixoto and M. Rocha, Coord. Chem. Rev., 2019, 394, 104.

4 (a) Y. Ren, J. Chen, C. Qi and H. Jiang, ChemCatChem, 2015, 7, 1535; (b) S. He, F. Wang, W.-L. Tong, S.-M. Yiu and M. C. W. Chan, Chem. Commun., 2016, 52, 1017; (c) J. A. Castro-Osma, M. North and X. Wu, Chem.-Eur. J., 2016, 22, 2100; (d) X. Wu, J. Mason and M. North, Chem.Eur. J., 2017, 23, 12937; (e) S. Vagin, M. Winnacker, A. Kronast, P. T. Altenbuchner, P. Deglmann, C. Sinkel, R. Loos and B. Rieger, ChemCatChem, 2015, 7, 3963; (f) W. Luo, T. Shi, S. Liu, W. Zuo and Z. Li, Organometallics, 2017, 369, 1736.

5 (a) S. J. Wezenberg, E. C. Escudero-Adán, J. Benet-Buchholz and A. W. Kleij, Org. Lett., 2008, 10, 3311; (b) J. Cheng, $\mathrm{X}$. Ma, Y. Zhang, J. Liu, X. Zhou and H. Xiang, Inorg. Chem., 2014, 53, 3210.

6 C. Bizzarri, E. Spuling, D. M. Knoll, D. Volz and S. Bräse, Coord. Chem. Rev., 2018, 373, 49.

7 A. K. Asatkar, S. P. Senanayak, A. Bedi, S. Panda, K. S. Narayan and S. S. Zade, Chem. Commun., 2014, 50, 7036.

8 G. Salassa, J. W. Ryan, E. C. Escudero-Adána and A. W. Kleij, Dalton Trans., 2014, 43, 210. 
9 (a) R. M. Clarke and T. Storr, Dalton Trans., 2014, 43, 9380; (b) M. Andruh, Chem. Commun., 2011, 47, 302; (c) P. Fuentealba, V. Paredes-Garcia, D. Venegas-Yazigi, I. D. A. Silva, C. J. Magon, R. C. de Santana, N. Audebrand, J. Manzurh and E. Spodine, RSC Adv., 2017, 7, 33305.

10 K. T. Mahmudov and A. J. L. Pombeiro, Chem.-Eur. J., 2016, 22, 16356.

11 (a) Y. S. Kudyakova, D. N. Bazhin, M. V. Goryaeva, Y. V. Burgart and V. I. Saloutin, Russ. Chem. Rev., 2014, 83, 120; (b) P. Subramaniam, C. Ramasubbu and S. Athiramu, Green Chem., 2017, 19, 2541.

12 (a) P. P. Mukovoz, P. A. Slepukhin, E. A. Danilova, O. P. Aysuvakova and A. P. Glinushkin, Russ. J. Gen. Chem., 2018, 88, 1363; (b) N. A. Bailey, K. C. Cox, C. P. Falshaw, D. E. Fenton, S. E. Grundy, P. Haigh, C. A. Phillips and T. J. King, Dalton Trans., 1983, 2241.

13 (a) D. E. Fenton and S. E. Gayda,J. Chem. Soc., Dalton Trans., 1977, 2095; (b) D. E. Fenton and S. E. Gayda, Dalton Trans., 1977, 2109; (c) C. K. Lai, A. G. Serrette and T. M. Swager, J. Am. Chem. Soc., 1992, 114, 7948; (d) R. P. Kreh, A. E. Rodriguez and S. P. Fox, J. Chem. Soc., Chem. Commun., 1984, 130.

14 (a) D. L. Obydennov, L. R. Khammatova, O. S. Eltsov and V. Y. Sosnovskikh, Org. Biomol. Chem., 2018, 16, 1692; (b) D. L. Obydennov, L. R. Khammatova and V. Y. Sosnovskikh, Mendeleev Commun., 2017, 27, 172; (c) D. L. Obydennov, E. O. Pan'kina and V. Y. Sosnovskikh, Chem. Heterocycl. Compd., 2017, 53, 924; (d) D. L. Obydennov, E. O. Pan'kina and V. Y. Sosnovskikh, J. Org. Chem., 2016, 81, 12532.

15 D. L. Obydennov and V. Y. Sosnovskikh, Chem. Heterocycl. Compd., 2014, 50, 579.

16 H. C. Kolb, M. G. Finn and K. B. Sharpless, Angew. Chem., Int. Ed., 2001, 40, 2004.

17 (a) V. S. Berseneva, A. V. Tkachev, Y. Yu. Morzherin, W. Dehaen, I. Luyten, S. Toppet and V. A. Bakulev, J. Chem.
Soc., Perkin Trans. 1, 1998, 2133; (b) V. S. Berseneva, Y. Y. Morzherin, W. Dehaen, I. Luyten and V. A. Bakulev, Tetrahedron, 2001, 57, 2179; (c) A. Ranise, F. Lucchesini, M. Caviglia, S. Alfei, A. Spallarossa and C. Caneva, Tetrahedron, 2013, 69, 10858; (d) J. Schreurs, C. A. H. van Noorden-Mudde, L. J. M. van de Ven and J. W. de Ham, Org. Magn. Reson., 1980, 13, 354.

18 (a) J. Saloň, V. Milata, A. Gatial, N. Prónayová, J. Leško, P. Černuchová, Z. Rappoport, G. Vo-Thanh and A. Loupy, Eur. J. Org. Chem., 2005, 4870; (b) J. Pigošová, A. Gatial, V. Milata, P. Černuchová, N. Prónayová, T. Liptaj and P. Matějka, J. Mol. Struct., 2005, 744-747, 315; (c) A. Gatial, S. Dorotíková, K. Plevová, V. Milata, N. Prónayová and P. Matějka, J. Mol. Struct., 2015, 1090, 112.

19 C. Hametner, P. Černuchová, V. Milata, G. Vo-Thanh and A. Loupy, Org. Magn. Reson., 2005, 43, 171.

20 A. Brbot-Šaranović, G. Pavlović and M. Cindrić, Struct. Chem., 2000, 1, 65.

21 (a) A. V. Pestov, L. A. Khamidullina, V. Y. Sosnovskikh, P. A. Slepukhin and I. S. Pusyrev, Polyhedron, 2016, 106, 75; (b) Y. Yu, J. Chen, S. Meng, C. Li, M. Lan and Z. Zhang, J. Mol. Catal. A: Chem., 2013, 380, 104; (c) T. Rogez-Florent, S. Meignan, C. Foulon, P. Six, A. Gros, C. Bal-Mahieu, C. T. Supuran, A. Scozzafava, R. Frédérick, B. Masereel, P. Depreux, A. Lansiaux, J.-F. Goossens, S. Gluszok and L. Goossens, Bioorg. Med. Chem., 2013, 21, 1451.

22 (a) J. H. Satcher and A. L. Balch, Inorg. Chem., 1995, 34, 3371; (b) D. L. Reger, A. E. Pascui, P. J. Pellechia and A. Ozarowski, Inorg. Chem., 2013, 52, 12741.

23 (a) V. Y. Sosnovskikh, A. V. Safrygin, R. A. Irgashev, M. A. Ezhikova and M. I. Kodess, RSC Adv., 2016, 6, 30056; (b) R. A. Irgashev, A. V. Safrygin, M. A. Ezhikova, M. I. Kodess, G.-V. Röschenthaler and V. Y. Sosnovskikh, Tetrahedron, 2015, 71, 1822.

24 D. L. Obydennov, A. O. Goncharov and V. Y. Sosnovskikh, Russ. Chem. Bull., 2016, 65, 2233. 